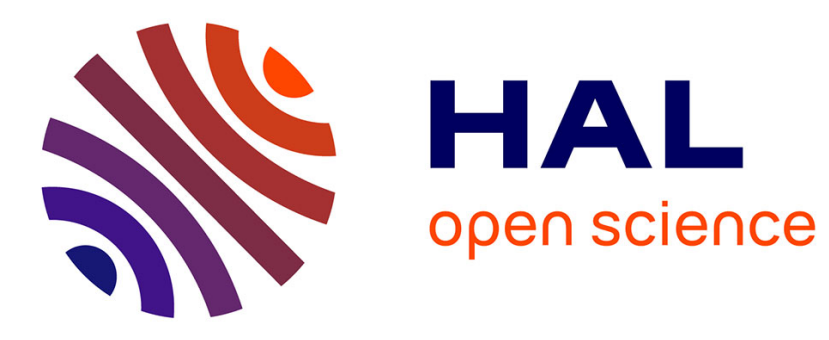

\title{
Un potier inconnu: P - I : remarques sur la détermination des officines anonymes
}

Colette Bémont, George B. Rogers

\section{To cite this version:}

Colette Bémont, George B. Rogers. Un potier inconnu: P - I : remarques sur la détermination des officines anonymes. Gallia - Fouilles et monuments archéologiques en France métropolitaine, 1980, 38 (1), pp.169-196. 10.3406/galia.1980.1793 . hal-01938976

\section{HAL Id: hal-01938976 https://hal.science/hal-01938976}

Submitted on 27 Feb 2020

HAL is a multi-disciplinary open access archive for the deposit and dissemination of scientific research documents, whether they are published or not. The documents may come from teaching and research institutions in France or abroad, or from public or private research centers.
L'archive ouverte pluridisciplinaire HAL, est destinée au dépôt et à la diffusion de documents scientifiques de niveau recherche, publiés ou non, émanant des établissements d'enseignement et de recherche français ou étrangers, des laboratoires publics ou privés.

\section{(ㅇ)(1) $\$$}

Distributed under a Creative Commons Attribution - NonCommercial - NoDerivatives| 4.0 


\title{
UN POTIER INCONNU : P-I \\ REMARQUES SUR LA DÉTERMINATION DES OFFICINES ANONYMES
}

\author{
par Colette BÉMONT et George ROGERS
}

Les enquêtes que nous menons depuis quelques années nous ont fait découvrir, à côté de produits de potiers bien connus de la Gaule centrale, des décors actuellement irréductibles au style de l'un ou l'autre de ces derniers, parfois identifiés grâce à une signature, le plus souvent anonymes. Selon la richesse ou la pauvreté iconographique

II) de nos documents, la nature du support et de l'ornementation, le nombre des témoins, nous avons soit défini ou redéfini des styles ${ }^{1}$, soit inventorié des individus ou de petits groupes en réservant la possibilité de leur attribution ultérieure ${ }^{2}$. Une série de vingt-six moules et vases anonymes s'était signalée à l'attention par la richesse de son répertoire, l'homogénéité structurelle d'une partie des compositions décoratives et l'emploi apparemment exclusif de divers poinçons secondaires ${ }^{3}$. Cet ensemble avait été classé sous la dénomination P-1. Un nouvel inventaire du matériel du Musée des Antiquités nationales (M.A.N.) de Saint-Germain-en-Laye nous a amenés à revoir la composition du groupe et à enrichir son répertoire de plusieurs motifs pour une part inédits. De plus, la chance, en nous faisant connaître récemment un fragment de moule de la même famille, mais estampillé, nous autorise désormais à associer un nom à ces produits.

La constitution d'un ensemble cohérent, lorsque ses références épigraphiques sont nulles (comme ce fut d'abord le cas) ou très peu nombreuses, ne peut se fonder que sur des caractères intrinsèques - c'est-à-dire principalement sur l'inventaire du répertoire, les multiples associations de poinçons, dont le jeu varie d'une frise à l'autre, le caractère

1 G. B. Rogers, Poteries sigillées de la Gaule centrale. I Les motifs non figurés, XXVIII Suppl. à Gallia, Paris, 1974 : les styles $P, M, X-0, X-8$ à $X-14$ (Avant-propos, p. 17 ; Principes du catalogue, p. 21 et 23); C. BÉmont, Moules de gobelets ornés de la Gaule centrale au Musée des Antiquités nationales, XXXIII Suppl. à Gallia, Paris, 1977, produits de Campanus, Florianus, Lucius, Florus, Sedatus.

2 C. BÉmont, o.c., p. 24-25 et 35.

3 Vases : 5 tessons de Biot (coll. Augé-Laribé, issue de la coll. Plicque), 4 du Musée des Antiquités nationales (coll. Plicque : P 1775, P 1777, P 1778, P 1779), 1 de Brecon. Moules : 16 tessons du II.A.N. (coll. Plicque : P.M 511 à 521,523 à 526, MAN 65995). 
remarquable voire exclusif de certains motifs et de leur assemblage. Aussi le problème principal est-il de s'assurer, malgré la possible diversité du contenu des décors, de la solidité des liens iconographiques qui unissent toutes les pièces de la série et, en revanche, de la faiblesse d'autres possibilités d'attribution.

En l'occurrence, notre attention a été attirée, d'abord, par l'emploi de deux oves (fig. 5,7 et 8 ) B 10 et $\mathrm{B} 203^{4}$ - associés à un nombre notable de poinçons secondaires, et de façon régulière, parfois exclusive ${ }^{5}$. En revanche, les répertoires des deux séries de décors ainsi constituées présentaient globalement tant de points communs qu'il nous a paru, au moins provisoirement, impossible de les disjoindre. Et, faute d'autre référence plus contraignante, nous avions admis dans un premier temps que les frises associées à chacune des deux bordures relevaient toutes d'un même atelier.

Cependant, nous ne pouvions nous dissimuler qu'une partie des décors liés à l'ove à gland, B 203, posait un problème. En effet, une série de six fragments ${ }^{6}$ de petite taille, ornés pour ce qui restait de rinceaux ou de festons végétaux, constituait un ensemble relativement clos (fig. 1, 2). Exclusifs $^{7}$, ou employés par un nombre réduit de potiers apparemment dispersés ${ }^{8}$, la plupart des poinçons les mieux déterminés ne semblaient associés entre eux que dans ce groupe, mais les liens possibles avec le reste des frises au même ove, ou avec l'ensemble du matériel que nous avions attribué alors au style $P-1$, paraissaient ténus et limités à de rares éléments géométriques souvent peu caractérisés : oves, cordons, arceaux, tortillons ${ }^{9}$. La suite de notre recherche nous a conduits à séparer cette série de $P$-1. Car la découverte de sept nouveaux tessons ornés du même répertoire végétal, parfois du même ove $\mathrm{e}^{\mathbf{1 0}}$, a confirmé la régularité des associations entre ces quelques poinçons spécifiques, mais aussi l'absence de liens significatifs entre les treize fragments et le reste de $P-1$ (fig. 1,3). L'emploi exclusif de l'ove B 203 devenait donc d'autant plus hypothétique. En outre le répertoire original de la série s'est accru de nouveaux éléments ${ }^{11}$. Enfin, et surtout, une relecture de certains motifs sur des exemplaires mieux conservés que les premiers témoins, ainsi que la prise en compte systématique des différences de taille possibles entre poinçons sur moules et sur vases, ont permis de relever un nombre non négligeable d'identités avec le répertoire et de ressemblances avec les compositions d'un autre groupe (fig. 1,1), composé de quelques échantillons homogènes et

4 G. B. Rogers, o.c., p. 40 et 47.

5 Poinçons tenus pour propres à $P-1$ : associés à B 10 : G 91, G 304, J 101, J 102, K 1, K 28, S 9, U 100 , U 188; associés à B 203 : C 234, G 286, G 322, J 114, S 17 ; associés aux deux oves : G 23, G 160, H 159, J 184, U 94, $\mathrm{U} 201$.

6 Biot 1-4; P 1775, P 1778.

7 J 114 : Biot $3 ; C 234$ : Biot $2 ; G 286:$ P 1775, Biot 2 el 5.

8 G $281: P-4, P-30, M E .$. (s) ; G $39: M-1(=P-4 ?) ; \mathrm{J} 9: P-4 ; \mathrm{J} 89: X-9, X-11$, potier à la Roselte, Criciro.

9 Ove B 203, cordon ondulé A 23, c. tireté A 14, arceau simple el tortillons non recensés. La plupart ders rosettes n'étaient pas lisibles et les imbrications - réduites aux pointes —, pas identifiables.

10 Il s'agit uniquement de vases, pour la plupart de la coll. Plicque : P 1720, 11734, 1760, 1794, 1797, MAN 49753 et 25623 (coll. Rambert). 49753 et P 1720 conservent seuls l'ove B 203, les autres fragments ne sont identifiés que par leurs poinçons végétaux exclusifs : J 114 (P 1734, 1760, 1794, 1797), G 286 (49753, 25623, P 1760), petit H 95 (49753), G 281 (P 1720) étant attribué à la fois à $P-1$ (Biot 1 ) et $P-4$.

11 Petit fleuron trifide peu lisible (P 1734, 1794, 1797), petite feuille à six lobes (P 1794, 1797), grappe de la Graufesenque (49753), grande spirale (P 1797), trombe striée (P 1760), auxquels s'ajoute un poinçon figuré : un aigle de face (P 1760). 

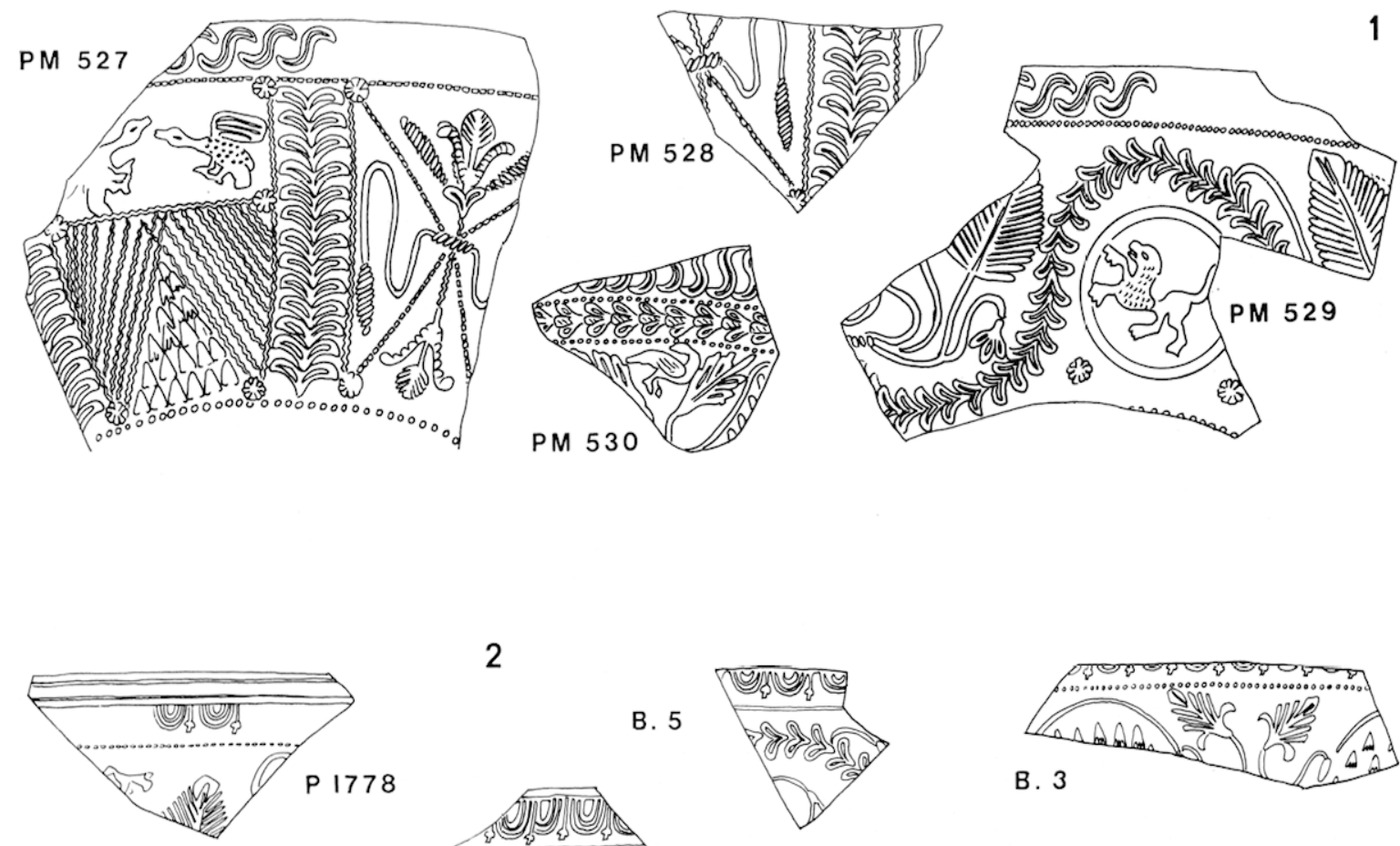

2
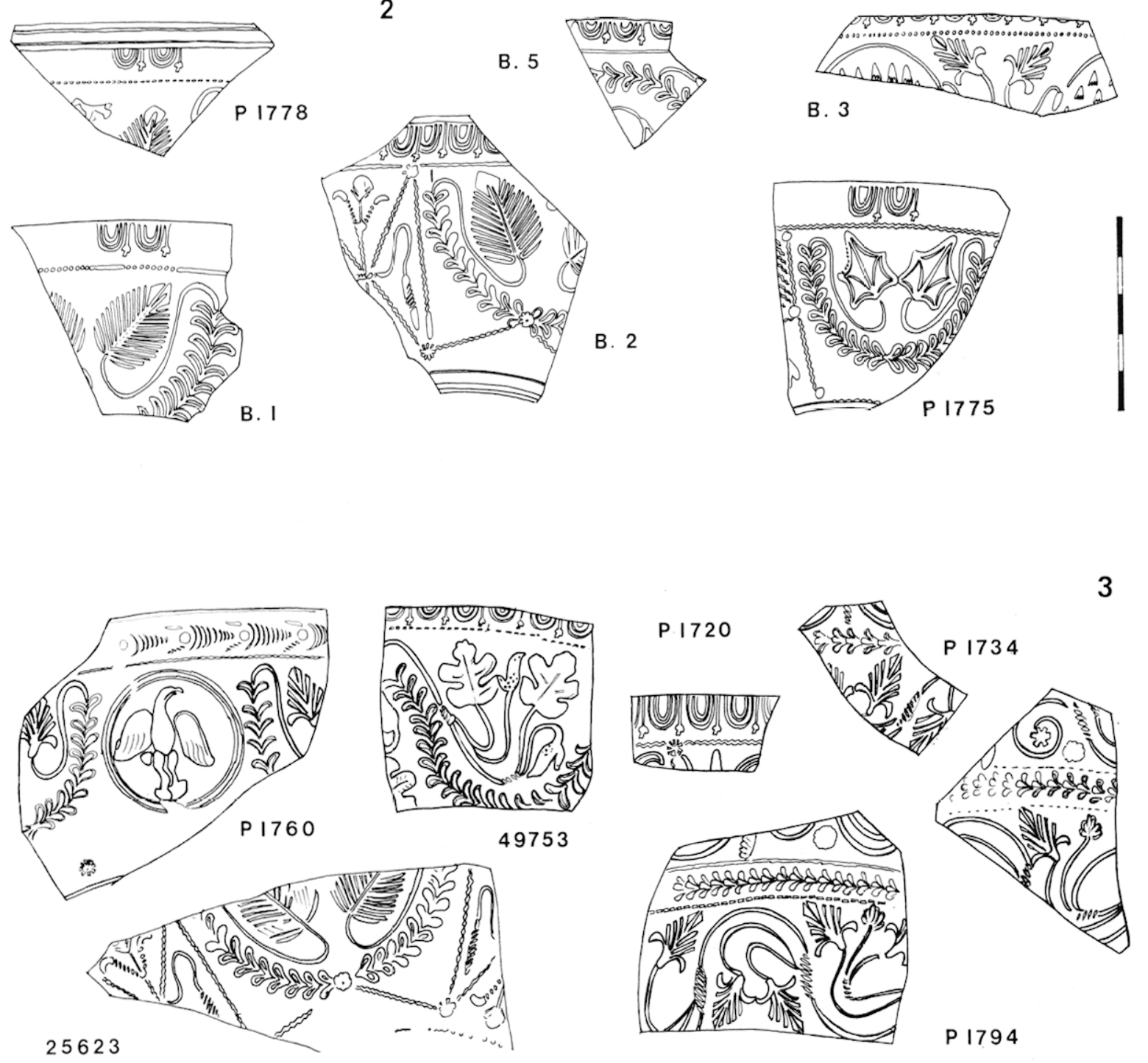

1 P.4. 1 : groupe primitif (moules du M.A.N., coll. Plicque); 2 : groupe d'abord attribué à $P$-1 (P 1775 et P 1778 : vases du M.A.N., coll. Plicque; B. 1-3, 5 : vases de Biot, fragment de la coll. Plicque); 3 : dernier groupe (vases du M.A.X. : coll. Rambert $(49753,25623)$ et Plicque). 
baptisé provisoirement $P-4^{12}$. Il nous a paru, dès lors, plus justifié d'inclure les tessons douteux dans ce dernier et de partager désormais, au moins, entre $P$ - 1 et $P$-4 l'usage de l'ove B $203^{13}$.

Furent-ils seuls à l'utiliser ? Parmi le reste des échantillons pareillement bordés un petit fragment de bol demeure totalement isolé : P 1777. Orné de deux métopes - meublées l'une d'un sautoir composite, l'autre d'un belluaire et d'un sanglier entre deux frises horizontales de chevrons --il se caractérise, du moins pour les poinçons bien lisibles, par les décors menus, les motifs étriqués qu'il est seul à porter (fig. 2). Toutefois, nous ne connaissons pas d'autre atelier auquel on pourrait l'attribuer.

A ce noyau principal, composé d'abord de dix moules et un ou deux vases, puis de douze moules et deux ou trois vases (fig. 6 à 9), ornés de l'un ou l'autre des deux oves ${ }^{14}$ se sont ajoutés, à l'occasion de découvertes successives, plusieurs échantillons qui répondaient, chacun à sa manière, aux mêmes exigences : avoir, toujours, avec le répertoire et, souvent, avec l'organisation des frises déjà rassemblées un très grand nombre de points communs - à la réserve toutefois des oves -, présenter dans tous les cas plus de ressemblances avec ce style qu'avec aucun de ceux que nous connaissons. Plusieurs fragments se sont intégrés sans problème majeur.

Certains - deux tessons de vases, un de moule ${ }^{15}$ - ont pour bordure des frises différentes des lignes d'oves (fig. 7). Les témoins d'un deuxième groupe portent, deux oves autres que B 10 et B 203. L'un d'eux (B 8) (fig. 5, 6), identifié depuis plusieurs années, est utilisé également dans un autre style anonyme - très différent par ses structures, mais apparenté par son répertoire -, baptisé $X-0^{16}$. Il caractérise en la circonstance deux moules ${ }^{17}$, le premier presque intact, le second très mutilé, mais quasi identique au précédent en sa partie conservée (fig. 6 , en bas). Un autre ove, apparemment inédit, figure sur un fragment de moule signé (fig. 5,$54 ; 7$ ), que nous a récemment communiqué un collectionneur ${ }^{18}$. La dernière série de tessons (un moule et quatre vases) est composée d'échantillons disparates et très mutilés ${ }^{19}$ qui se rattachent par la totalité ou la grande majorité de leurs poinçons au répertoire de $P-1$ (fig. 6, Brecon, $P 1791 ; 7, P M 524$ et $49695 ; 9, P 1742$ ), voire, pour au moins deux d'entre eux, à certaines de ses structures décoratives ${ }^{20}$.

A la différence des précédentes séries un tesson de moule et les fragments de deux décors de vases ont soulevé des difficultés.

Le premier échantillon, PM 526, porte quatre poinçons figurés et quatre détails secondaires attestés chez $P$-1 (fig. 2). En revanche, il présente deux originalités : le motif de remplissage U 93

12 Le fleuron G 39 sur vase correspond apparemment à G 38 sur moule $P$-4 ; le chevron $G 286$ est déformé à la base par la superposition du poinçon suivant, mais, détaché sur le tesson P 1760, il s'identifie en fait à G 284 (décor de $P$-4 plus grand parce qu'imprimé sur moule). Enfin, on peut reconnaitre sur $P 1720, P 1760$ et Biot 2 la rosette à neuf pétales inégaux de $P-4:$ C 195 .

13 Le répertoire de $P-1$ se trouve de ce fait amputé des poinçons C 234, H 95, G 39, G 281, G 286, J 9, J 89, J 114.

14 Ove B 10 : moules PM 513 à 518, auxquels s'est ajouté le vase P 1191 ; ove B 203 : moules PM 519 à 521 , PM 523, auxquels se sont ajoutés PM 522 et MAN 32419, vases P 1777 ? et 1779.

15 Moule PM 511, vases P 1780 et 25739 (coll. Rambert).

16 Poinçons secondaires communs aux deux styles : A 22, A 23, B 8, C 225, G 290, G 298, G 338, S 19, S 54, U 93, U 187.

17 MAN 65995 et PM 512.

18 Nous remercions M. Hervé Brun de l'amabilité avec laquelle il nous a permis de faire létude de ce fragment.

19 Moule PM 524, fragments de bols 49695, 1742, 1791 et de Brecon.

20 P 1791 et vase de Brecon; il n'est pas exclu même que P 1791 soit issu du moule MAN 32419. 


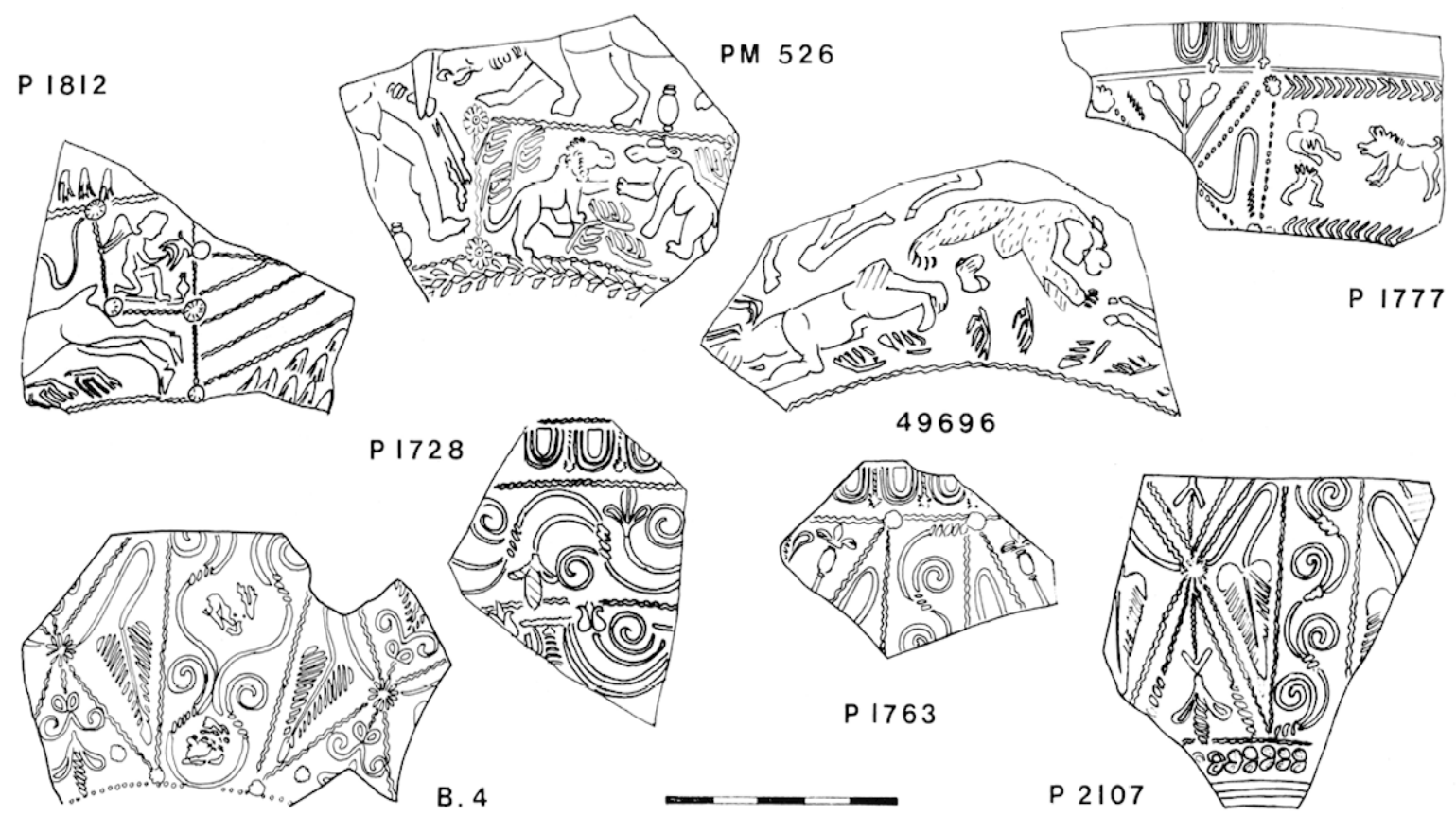

2 Échantillons douteux (coll. Plicque). P 1777 el P.M 526 : vase et moule du M.A.N. sans autre attribution que $P-1$; P 1812 et 49696 : vases non identifiés du M.A.N. portant le poinçon U 93 . En bas : tessons de bols écartés du style P-1 (vases de Biot : B. 4, et du M.A.N.).

(fig. 5,46 ) et une métope découpée, telle une pièce, à l'intérieur d'un grand panneau. Or le remplissage plusieurs fois utilisé sur nos échantillons $P-1$ (fig. 7) est U $94^{21}$. Quant à U 93 , il apparaît aussi dans le style $X-0$, qui n'offre, par ailleurs, aucune comparaison plus satisfaisante pour PM 526. Toutefois, nous connaissons maintenant, au M.A.N. deux fragments de bols, sans attribution certaine, qui portent le même petit décor associé à différents animaux et à un putto ; l'un d'eux comporte aussi une découpe à l'intérieur d'un panneau (fig. 2). Malheureusement aucun des animaux ne se retrouve dans les répertoires actuellement connus de $P-1$ ou $X-0$. Les particularités de PM 526 et l'extension probable de l'emploi de U 93 commandent donc une certaine réserve pour l'attribution du tesson à $P-1$, mais aucune autre solution positive ne s'impose.

Le dernier groupe d'échantillons pose d'une autre manière le problème de la valeur respective de deux critères d'identification : la structure et le répertoire.

En effet, nous avons inclus un fragment de Biot (fig. 2, B.4), dans le premier inventaire des produits $P-1$, en nous fondant sur l'aspect très particulier d'un décor de métope : deux rinceaux nus, verticaux, affrontés et meublés de petits motifs de remplissage. Cette composition est apparue jusqu'à présent seulement dans trois frises attribuées à $P-1$ (fig. 7 et 8), où elle est complétée par de petits masques : deux moules ornés de l'ove $B \mathbf{1 0}$ et le fragment signé22. Elle se retrouve sur le tesson de Biot, meublée d'un cerf minuscule connu déjà chez le potier à la Rosette ${ }^{23}$. Par ailleurs, le panneau

21 Moules MAN 32419, PM 514, PM 524, tesson signé.

22 Moules PM 513 et 515.

23 Cf. J. A. Stanfiel.d et G. Simpson, Central Gaulish Polters, Oxford, 1958 (abrégé : S.S.), pl. 20, $258=$ D 848. F. Oswald (Index of Figure Types, $2^{\mathrm{e}}$ éd., Londres, 1964, $\mathrm{n}^{\circ} 1702 \mathrm{~A}$ ) donne de ce cerf une image grossie sans raison apparente. 
au cerf est entouré sur ce bol de deux sautoirs composites dont les poinçons nous ont paru d'abord soit nouveaux - J 33, G 169, 290 et 375, rosette à douze pétales - soit très proches de décors de $P-1$ - A 23, S 19 et 54. Or un nouvel inventaire nous a fait découvrir en deux exemplaires au moins $^{24}$, un fragment de frise de structure analogue (fig. 2 , en bas) - mais à rinceau simple - et qui rassemblait la plupart des nouveaux poinçons de Biot (J 33, G 169 et 290, la roselle), le cordon ondulé (A 23) de $P$-1, plusieurs décors semblables, au premier abord à des motifs de $P$ - 1 (spirales $\mathrm{S} 19$ et 54 , bouton G 232, chevron G 323) et un ove peu lisible, mais proche de B 5925. Cependant un examen attentif a, en premier lieu, révélé que le bouton éclaté, nettement imprimé, était différent du poinçon $P-1$. Mis en alerte, nous avons constaté alors que les spirales du rinceau, exactement comparables par leur forme aux modèles S 19 et 54, étaient de même taille sur ces tessons de vases que les spirales sur moules de $P-1^{26}$. Enfin, le moule signé imputé avec le maximum de vraisemblance au style $P-1$, portait une palme du type de $J 33$, mais de proportions différentes (fig. 7 ). Ainsi, attribuer le tesson de Biot à $P-1$ impliquait que le même potier ait utilisé, pour produire des décors analogues (sautoirs et rinceaux), deux séries de poinçons très proches mais pourtant distinctes. Compte tenu aussi des nouveautés apportées par ce fragment et le second bol, on pouvait donc se demander s'il ne s'agissait pas plutòt de deux potiers se copiant partiellement l'un l'autre et s'il ne fallait pas considérer les différences de répertoire de préférence à l'identité de mise cn œuvre. Une fois posée cette hypothèse, le petit cerf pouvait être tenu non pour une fantaisie de $P-1$, renonçant pour une fois aux masques, mais pour le différent choisi par l'émule de ce potier. En réalité, d'autres comparaisons ont révélé une parenté mieux caractérisée entre nos fragments et les produits de $X-11$ - B 59 ?, G 170, G 375 -, ou du potier à la Rosette - A 23, G 169, petit cerf -. Et un morceau de bol du M.A.N. (P 1728) (fig. 2) a confirmé l'association du même ove avec A 23 et S 19 et des poingons appartenant à ces deux répertoires (G 100) ou propres à $X-11$ (G 151, M 41). Il nous a donc paru sage d'écarter tous ces échantillons.

Au terme des critiques successives qui nous ont conduits à revoir l'idée première que nous nous faisions du style $P-1$, le groupe des produits les plus sûrs comprend vingt-six à vingt-huit bols D. 37 : dix-huit ou dix-neuf moules et huit ou neuf vases (fig. 6 à 9). Par ailleurs, treize tessons tous ornés principalement de motifs végétaux ont été attribués à $P-4$, tandis que les restes de quatre bols - décorés surtout de sautoirs et correspondant probablement à deux moules - constituent un groupe encore indéterminé. Le répertoire global de l'ensemble ainsi constitué comprend 49/53 poinçons secondaires recensés déjà, 27 autres non répertoriés (nouveaux ou appartenant à des catégories volontairement négligées comme peu significatives), 28 personnages ou animaux connus et $5 / 7$ inédits (fig. 3 à 5). La comparaison des listes de poinçons associés à chacun des oves permet de mettre en évidence les points communs entre les différentes séries et le tableau II ci-joint (p. 180), montre que, malgré des disparités que crée le nombre variable des échantillons, les groupes déterminés d'après ce critère entretiennent de multiples relations. Comme il est naturel, les sérics 1 ct 2 (oves B 10 et B 203), les plus nombreuses, sont aussi les plus étroitement associées, mais on notera également que la première fait le lien entre toutes les autres, dans la mesure où par son répertoire elle est la plus proche de chacune

24 Deux fragments d'un même bol trouvés dans les fouilles d'Augst (E. Ettúngé, Die Keramik der Augster Thermen, Bâle, 1949, pl. 47 et p. $71, n^{\circ} 225$ ) et deux tessons appartenant semble-t-il à deux tirages différents du même moule : P 1763 et 2107 (coll. Plicque).

25 Ove attribué jusqu'à présent à $X-11$.

26 Le chevron très usé de l'exemplaire du M.A.N. (P 2107) ne peut être identifié sùrement avec G 321 ( $X-8$ et $X-9)$ plutôt qu'avec G $323(P-1$ et plusieurs autres potiers). 
I. Inventaire du répertoire $P-1$

\begin{tabular}{|c|c|c|c|c|c|c|}
\hline N.I. & 0. & D. & N.I. & R. & N.I. & R. \\
\hline \multirow{14}{*}{$\begin{array}{l}\text { } \\
1 \\
1 \\
1 \\
1\end{array}$} & 12 & 13 & Ms 1 & A 1 & Ms 41 & S 19 \\
\hline & 28 & 25 & 2 & A $14 ?^{*}$ & 42 & S 38 \\
\hline & 233 & 145 & 3 & A 15 & 43 & S 39 \\
\hline & $306 \mathrm{~A}$ & & 4 & A 22 & 44 & S 40 \\
\hline & 339 & 193 & 5 & A 23 & 45 & S 54 \\
\hline & 433 & 227 & 6 & В 8 & 46 & U $93^{\star}$ \\
\hline & 580 & 331 & 7 & B 10 & 47 & U 94 \\
\hline & 723 & & 8 & B 203 & 48 & U 100 \\
\hline & 740 & 427 & 9 & C 132 & 49 & U 103 \\
\hline & $760 ?$ & & 10 & C 225 & 50 & U 187 \\
\hline & 860 & & 11 & C 229 & 51 & U 188 \\
\hline & putto & & 12 & E 24 & 52 & U 201 \\
\hline & belluai & & 13 & F 74 & 53 & $\mathrm{U} 202^{\star}$ \\
\hline & & & 14 & G 1 & 54 & ove \\
\hline \multirow{2}{*}{$\mathrm{Ma}$} & 1287 & 685 & 15 & G 23 & 55 & double cercle \\
\hline & 1313 & 710 & 16 & G 91 & 56 & anneau \\
\hline \multirow{24}{*}{$\begin{array}{c}\text { An } 1 \\
2 \\
3 \\
3 \\
4 \\
5 \\
6 \\
7 \\
8 \\
9 \\
10 \\
11 \\
12 \\
13 \\
14 \\
15 \\
16 \\
17 \\
18 \\
19 \\
20\end{array}$} & 1380 & 739 & 17 & G 101 & 57 & petit anneau \\
\hline & 1407 & 745 & 18 & G 144 & 58 & petit arceau \\
\hline & 1428 & 758 & 19 & G 160 & 59 & arceau \\
\hline & $1497 \mathrm{E}$ & & 20 & G $180 ?$ & 60 & grand arceau \\
\hline & $1497 \mathrm{~L}$ & & 21 & G 232 & 61 & arceau double \\
\hline & 1502 & 784 & 22 & G 252 & 62 & fleuron \\
\hline & 1607 & 815 & 23 & G 298 & 63 & fleuron \\
\hline & 1610 & $969 b$ & 24 & G 299 & 64 & chevron \\
\hline & 1613 & 955 & 25 & G 304 & 65 & grand chevron \\
\hline & 1733 & 856 & 26 & G $322^{\star}$ & 66 & palme \\
\hline & 1941 & 914 & 27 & G 323 & 67 & rameau à gauche \\
\hline & 2070 & 940 & 28 & G 338 & 68 & petit rameau à gauche \\
\hline & $2134 ?$ & & 29 & G 349 & 69 & petit rameau à droite \\
\hline & $2184 ?$ & 980 & 30 & H 108 & 70 & touffe fleurie \\
\hline & 2292 & 1036 & 31 & Н 159 & 71 & touffe d'herbe \\
\hline & \multirow{5}{*}{\multicolumn{2}{|c|}{$\begin{array}{l}\text { lion à droite } \\
\text { sanglier à gauche } \\
\text { gazelle à gauche } \\
\text { biches ? à droite } \\
\text { dragon }\end{array}$}} & 32 & J 101 & 72 & grande spirale \\
\hline & & & 33 & J 102 & 73 & amphorette \\
\hline & & & 34 & J 184 & 74 & petite cruche \\
\hline & & & 35 & K 1 & 75 & tronc \\
\hline & & & 36 & K 28 & 76 & bâton torsadé \\
\hline & & & 37 & K 42 & 77 & pendentif torsadé \\
\hline & & & 38 & S 9 & 78 & pendentif torsadé \\
\hline & & & 39 & S 10 & 79 & mince tortillon \\
\hline & & & 40 & S 17 & 80 & fuseau torsadé \\
\hline
\end{tabular}

\section{Abréviations}

N.I. : numéro d'inventaire (planches)

O. : F. Oswald, o.c.

D. : J. Déchelette, Les vases céramiques ornés de la Gaule romaine, Paris, 1904

R. : G. B. Rogers, o.c.

$\mathrm{Pe} \quad$ : personnages

$\mathrm{Ma}:$ masques

An : animaux

Ms : motifs secondaires

* : poinçons appartenant exclusivement à des tessons douteux. 

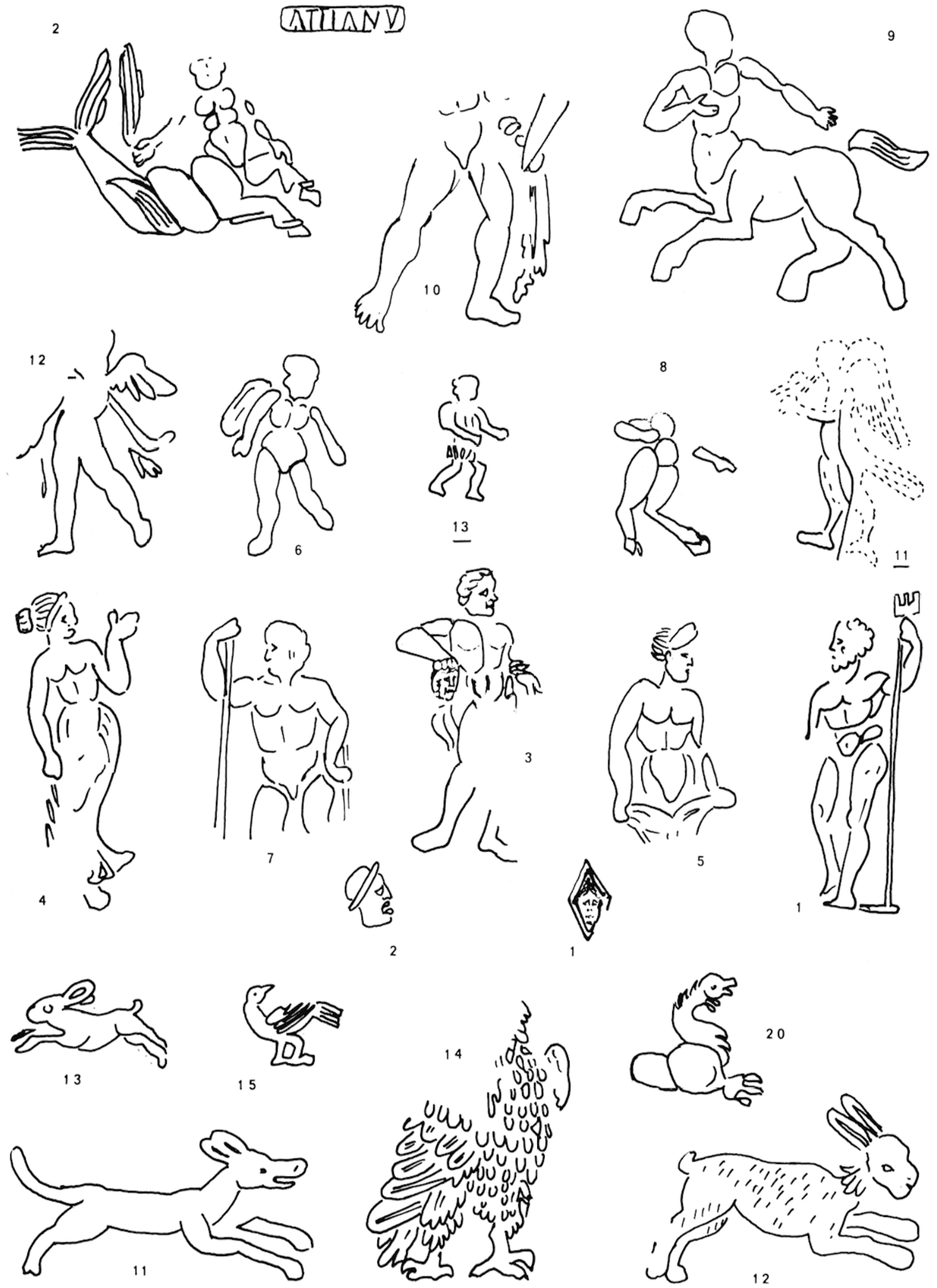

$3 \quad P$-1. Répertoire des poinçons (éch. 1:1). Estampille. Personnages (Pe). Masques (Ma). Animaux (An). Souligné : décor sur vase. 

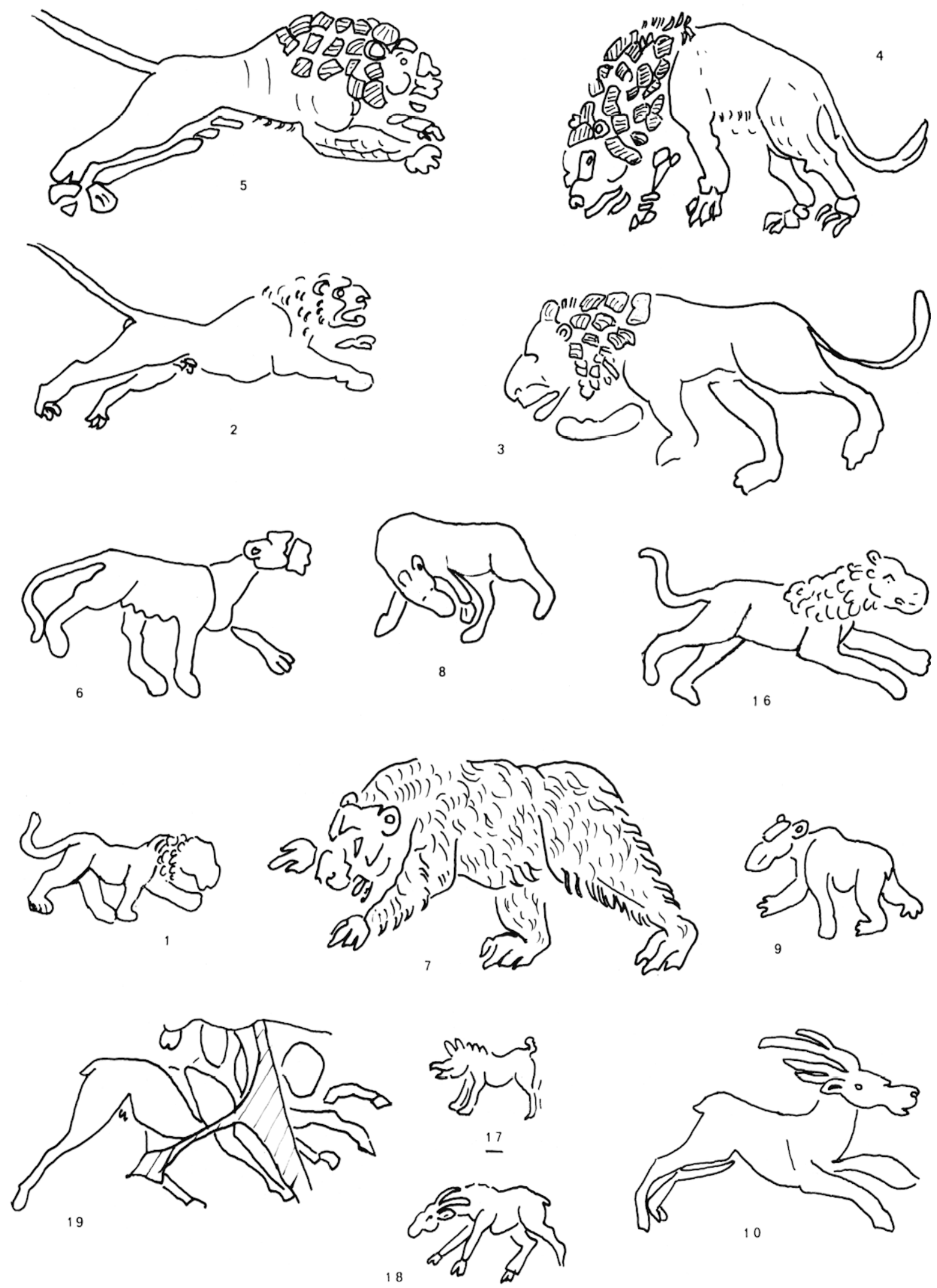

4 P-1. Répertoire des poinçons (éch. 1:1). Animaux (An). Souligné : décor sur vase. 


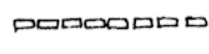

(U) (1)
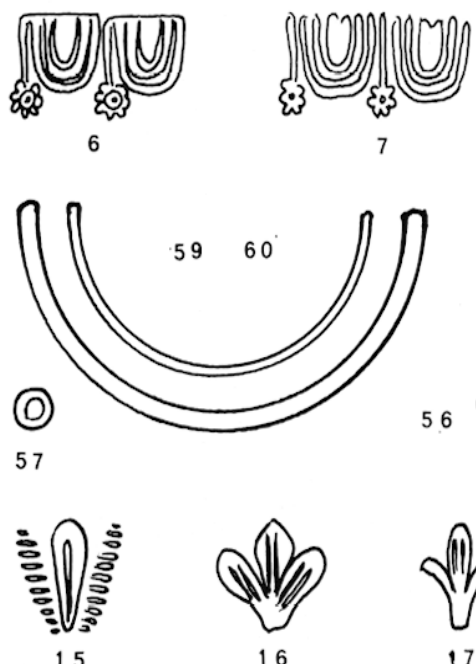

,

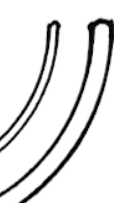

${ }_{56}$ (O)
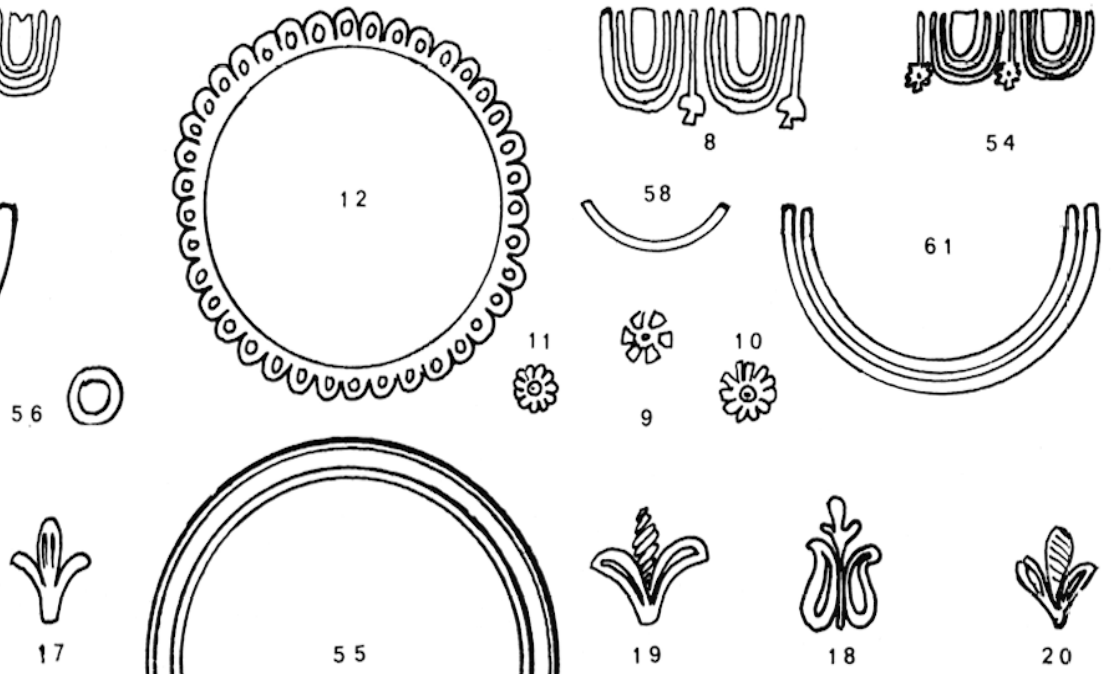

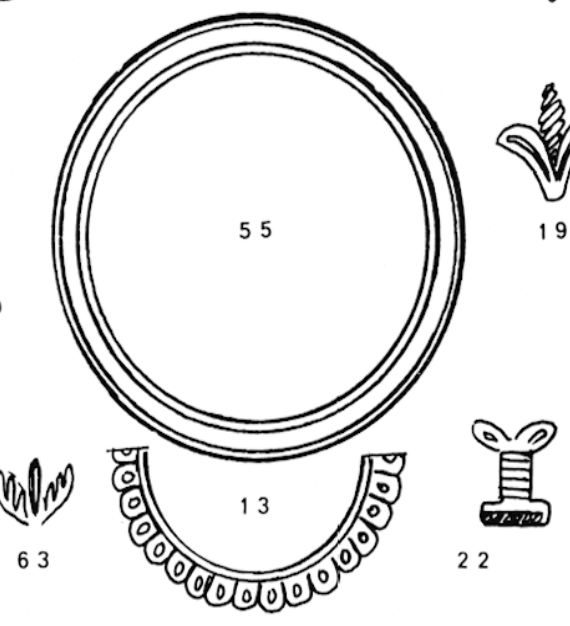

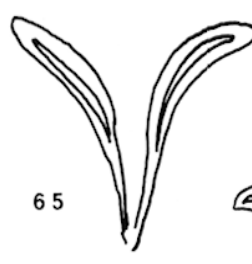

$\approx$

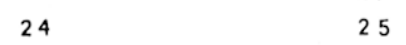
थ

N 26
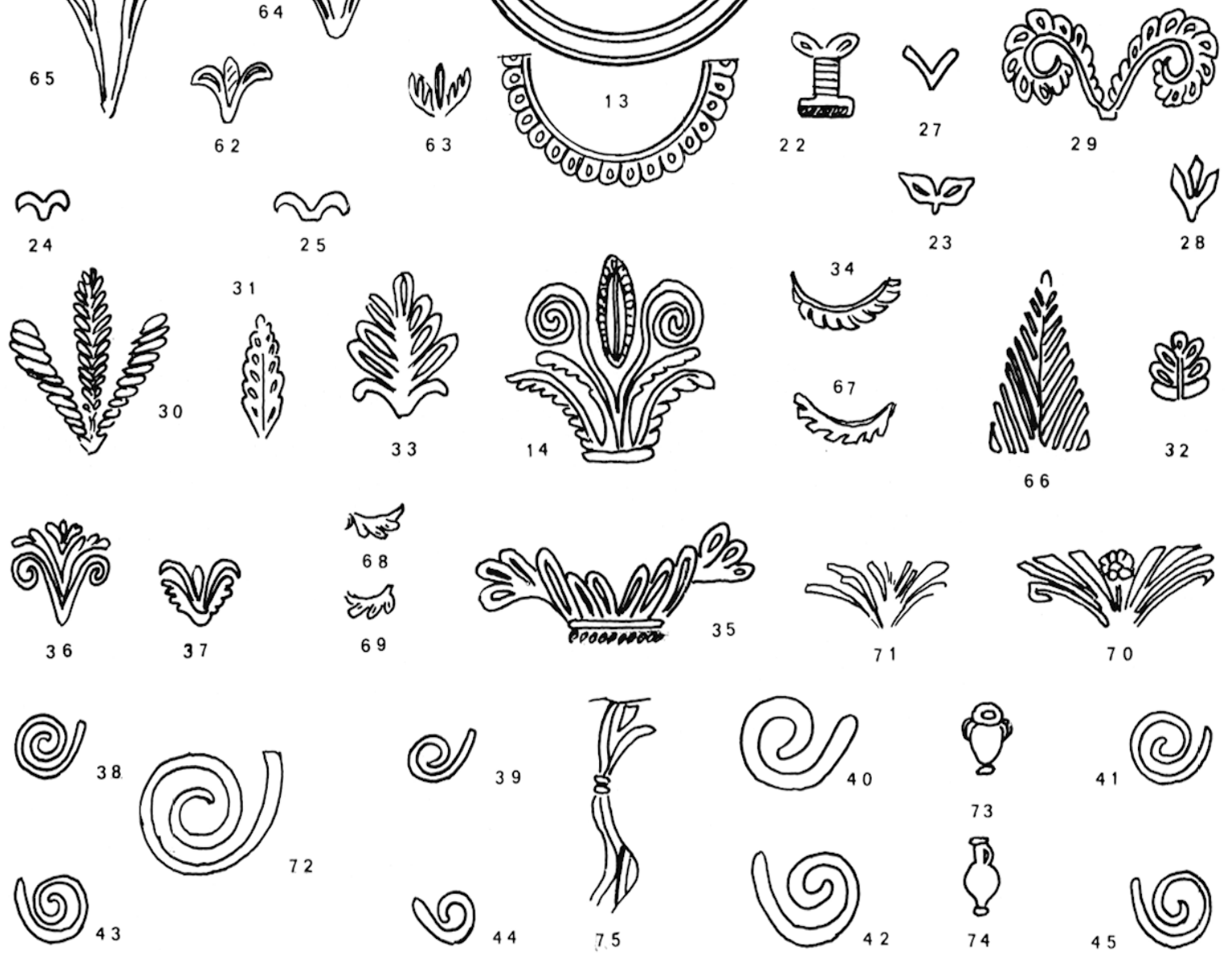

(2)) 


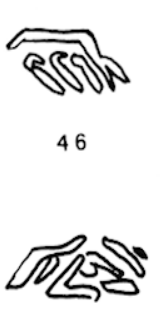

47

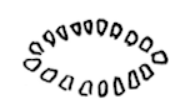

48

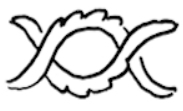

49
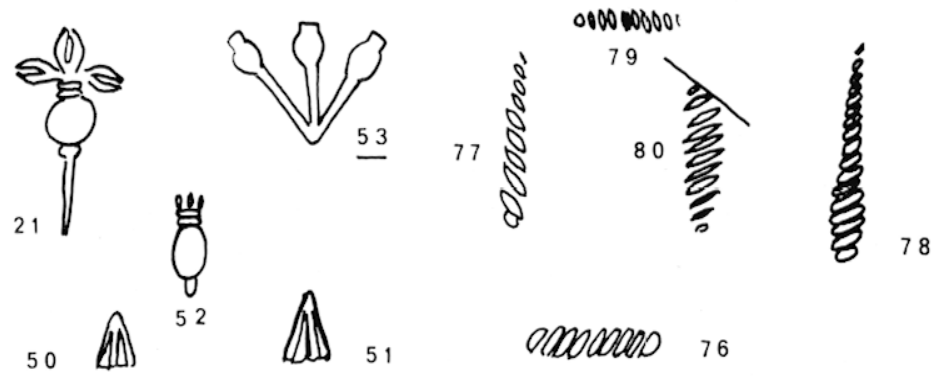

OCDOOOODO 76

$5 \quad P$-1. Répertoire des poinçons (éch. 1:1). Motifs secondaires (Ms). Soulignés : décors sur vase.

d'elles. Cette unité explique sans doute qu'il soit impossible, d'après l'inventaire des poinçons, de rapprocher d'une série plutôt que d'une autre plusieurs des tessons dépourvus d'oves ${ }^{27}$. En revanche, nous ne saurions, compte tenu du petit nombre des échantillons, de leur état très inégal de conservation et de la profusion des décors (souvent originaux) figurant sur les témoins les mieux sauvegardés, considérer actuellement comme significative la présence exclusive de différents poinçons dans tel ou tel des groupes ${ }^{28}$ : une information moins lacunaire réduirait sûrement pour une bonne part ces disparates dues, le plus souvent, au mobilier d'un seul individu ${ }^{29}$.

La composition des frises, quand on peut l'analyser, révèle également certains facteurs d'unité.

Frises non géométriques. Ce sont les plus rares. Elles peuvent appartenir à des compositions à un seul registre ou à des décors à deux zones séparées par une ligne de fleurons. La première catégorie est illustrée par un tesson de moule à oves B 10 (PM 514), meublé, pour l'essentiel, de petits monstres, la seconde, par un fragment - peuplé aussi de monstres - de la partie inférieure d'un moule à deux registres (PM 524). Aucun des morceaux ne conserve de traces de division verticale ni ne permet de connaitre le rythme de répétition des poinçons (fig. 7).

\section{Frises à structures géométriques.}

Frises à découpage uniforme (fig. 9). Elles appartiennent toutes aux constructions à bandes superposées - caractéristiques de la transition entre les formes D. 29 et D. 37 et sont associées, au moins, à l'ove B 203. Elles comportent dans le meilleur cas (PM 521) deux zones de festons réguliers, tandis que deux autres échantillons ne conservent que

27 Moule PM 511, tessons de bols MAN 25739, 49695, P 1780 ; vase de Brecon.

28 Associés à l'ove B 10: A 1, C 132, G 91, G 298, J 101, Ms 57, O 740, Pe 12, O 1610, O 1941, O 2070, An 16, et Ms 62, O 28, O 724, An 20, si l'on intègre dans cette série, à cause de ses affinités, le tesson PM 524. Associés $\dot{a}$ l'ove B 203 : G 144, G 180 ?, G 349, H 108, S 17, Ms 56, Ms 61, Ms 63-65, Ms 72, o 306, O 580, O 1407, O 2134, O 2184, An 18, An 19; A 14 ?, G 322, U 202, Pe 13 et An 17, si l'on admet le tesson douteux P 1777, et Ms 55, Ms 70, Ms 71, O 1607, si l'on intègre dans la série les fragments P 1742 et 1791 ; enfin, S 38, F 74 et Ms 76 , si l'on intègre P.l 525. Associés à l'ove B 8: E 24, G 252, S 10, S 40, O 2292.

29 Par exemple le moule signé apporte à lui seul neuf nouveaux poinçons : O $12,01497 \mathrm{E}$, Ms 54 , Ms 66 , Ms 68, Ms 69, Ms 73, Ms 80, ainsi que Ms 74 qui permet d'identifier un détail incomplet du bol de Brecon ; MAN 32419 fournit neuf poinçons exclusifs: G 180 ?, G 349, Ms 64, Ms 65, Ms 77, o 1407, o 2134, An 18, An 19. 
II. Répartition des poinçons associés à plusieurs types de bordures

\begin{tabular}{|c|c|c|c|c|c|}
\hline N.P. & 1 & 2 & $\mathrm{~S}$ & 3 & 4 \\
\hline $\begin{array}{ll}\text { A } & 23 \\
\text { C } & 225 \\
\text { J } & 184 \\
\text { Ms } & 67 \\
\text { S } & 19 \\
\text { G } & 338 \\
\text { U } & 94 \\
\text { Ms } & 79 \\
\text { O } & 339 \\
\text { K } & 1 \\
\text { O } & 1502 \\
\text { A } & 15 \\
\text { G } & 299 \\
\text { S } & 54 \\
\text { U } & 187 \\
\text { K } & 28 \\
\text { C } & 229 \\
\text { G } & 23 \\
\text { G } & 101 \\
\text { G } & 160 \\
\text { H } & 159 \\
\text { K } & 42 \\
\text { U } & 201 \\
\text { Ms } & 78 \\
\text { O } & 233 \\
\text { O } & 1287 \\
\text { O } & 1497 \mathrm{~L} \\
\text { G } & 232 \\
\text { G } & 304 \\
\text { U } & 100 \\
\text { Ms } & 58 \\
\text { O } & 1313 \\
\text { S } & 9 \\
\text { S } & 39 \\
\text { O } & 1733 \\
\text { G } & 1 \\
\text { U } & 103\end{array}$ & $\begin{array}{l}0 \\
0 \\
0 \\
0 \\
0 \\
0 \\
0 \\
0 \\
0 \\
0 \\
0 \\
0 \\
0 \\
0 \\
0 \\
0 \\
0 \\
0 \\
0 \\
0 \\
0 \\
0 \\
0 \\
0 \\
0 \\
0 \\
0 \\
0 \\
0 \\
0 \\
0 \\
0 \\
0 \\
0 \\
0 \\
0\end{array}$ & $\begin{array}{l}\bullet \\
\bullet \\
0 \\
0 \\
? \\
\bullet \\
0 \\
0 \\
\bullet \\
\bullet \\
\bullet \\
\bullet \\
\bullet\end{array}$ & $\begin{array}{l}\bullet \\
\bullet \\
0 \\
0 \\
\bullet \\
\bullet \\
\bullet \\
\bullet \\
\bullet \\
\bullet\end{array}$ & $\begin{array}{l}\bullet \\
\bullet \\
\bullet \\
\bullet\end{array}$ & $\bullet$ \\
\hline $\begin{array}{ll}O & 1380 \\
O & 1613 \\
\text { Ms } & 74 \\
O & 433 \\
O & 760 ? \\
O & 1428\end{array}$ & $\bullet$ & $\begin{array}{l}0 \\
? \\
? \\
? \\
? \\
? \\
?\end{array}$ & 0 & $\bullet$ & 0 \\
\hline
\end{tabular}

Sigles el abrévialions

N.P. : numéro du poinçon

1 : série portant l'ove B 10

2 : série portant l'ove B 203

3 : série portant l'ove B 8

4 : série à bordure végétale

S : moule signé

- : poinçon secondaire ou type figuré actuellement exclusif

I, ? : attribution incertaine, faute de bordure, à une série. 
la frise géométrique supérieure (PM 522) ou inférieure (PM 525) et une part de la ligne de fleurons séparant les deux zones.

Frises à séquences de métopes. Les mieux conservées se classent en deux variétés principales. L'une, illustrée par au moins cinq moules plus ou moins mutilés et un tcsson de vase, se caractérise par une organisation foisonnante (fig. 6 et 7), remarquable surtout par la multiplicité des petits poinçons végétaux utilisés en semis ou en motifs complexes ${ }^{\mathbf{3 0}}$. La construction d'ensemble, lorsqu'elle est intelligible, se fonde sur un découpage du décor en huit grandes métopes organisées en deux séquences identiques de quatre panneaux différents entre eux (fig. 6, 65995). Le contenu des échantillons moins bien conservés est compatible avec ce principe d'organisation (fig. 6, PM 516 et 32419 en particulier). Ces métopes sont séparées les unes des autres par d'autres, très étroites et meublées de motifs composites, souvent touffus - doubles rinceaux, frises végétales, colonnes d'anneaux et le plus fréquemment divers pour un même décor. Ce mode de composition est associé à tous les oves sans qu'on puisse, étant donné le petit nombre des témoins, apprécier la signification des différences d'un exemplaire à l'autre.

$\mathrm{Du}$ fait de l'échantillonnage très inégal des groupes liés à chaque ove, la seconde variété de décors à métopes ne se rencontre qu'avec les types B 10 et B 203 (fig. 8 et 9). Beaucoup plus difficile à saisir dans son organicité à cause de l'état du matériel, elle présente essentiellement deux caractères distinctifs : la plus grande légèreté du décor et l'emploi, comme motifs de remplissage, autour des personnages ou animaux, de fleurons ou de boutons attachés aux angles des grands panneaux par de longues tiges souples. Ces mêmes tiges se retrouvent d'ailleurs - en concurrence avec les rinceaux, les frises végétales ou les files d'anneaux - dans de petites métopes séparant souvent les panneaux principaux (fig. 9). Sur l'échantillon le mieux conservé, PM 513, la composition paraît comparable, par le rythme des métopes et la variété de leur aménagement, au système de la précédente catégorie (fig. 8). Toutefois, d'autres bols malheureusement très mutilés témoignent d'organisations différentes : sur PM 520 les sautoirs composites abondent, et alternent régulièrement, du moins dans le plus grand fragment de décor, identiques entre eux, avec de hauts panneaux étroits occupés chacun par un personnage (fig. 9); un fragment de bol (P 1742), découpé en trois panneaux - l'un au centre avec un ours, les deux autres occupés chacun par un médaillon feuillu -, témoigne également de la même tendance aux répétitions fréquentes et mécaniques (fig. 9). La même monotonie reparaît dans l'occupation des panneaux de séparation : aucun échantillon, en effet, n'atteste encore l'emploi de plus d'un type de décor par vase. Dernier trait enfin : l'absence, sur certains témoins, de l'alternance régulière des grandes métopes et des colonnes de séparation (fig. 8, P 1779). L'extension totale et la variété réelle de cet ensemble stylistique demeurent cependant difficiles à déterminer, car le matériel se limite trop souvent à des fragments de décors certes aérés, mais dont la place dans une organisation globale nous échappe encore.

30 Moules MAN 32419 et 65995, PM 512 et 516 ; vase de Brecon. Le tesson de bol P 1791, s'il est issu du moule 32419, s'ajoute à ce groupe, mais n'apporte rien de neuf à la connaissance des décors. 


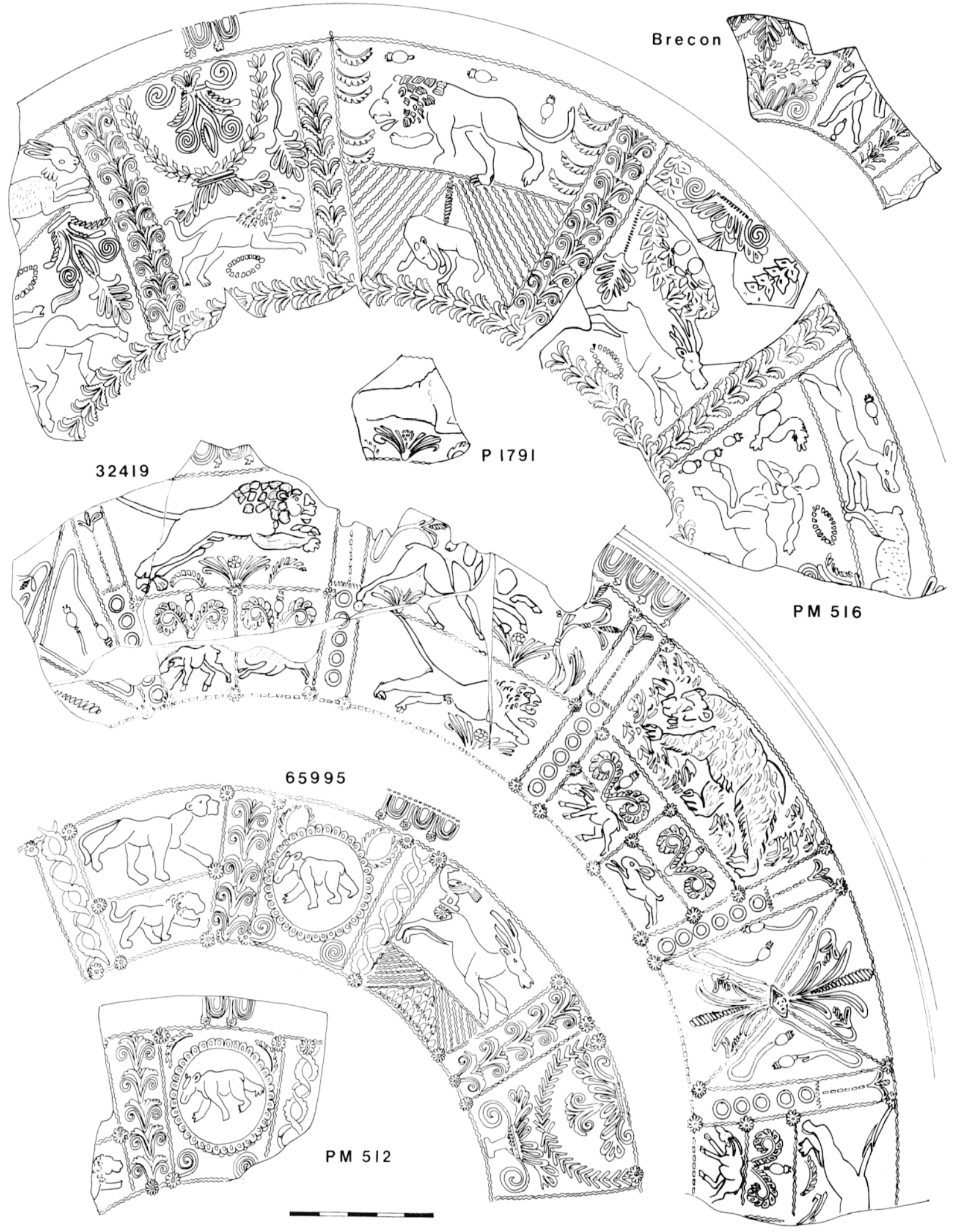

$6 \quad$ P-1. Style fleuri à métopes : vase (P 1791) et moules D. 37 du M.A.N. (coll. Plicque); fragment de bol de Brecon (d'après R. E. M. Wheeler, o.c., fig. 76). 


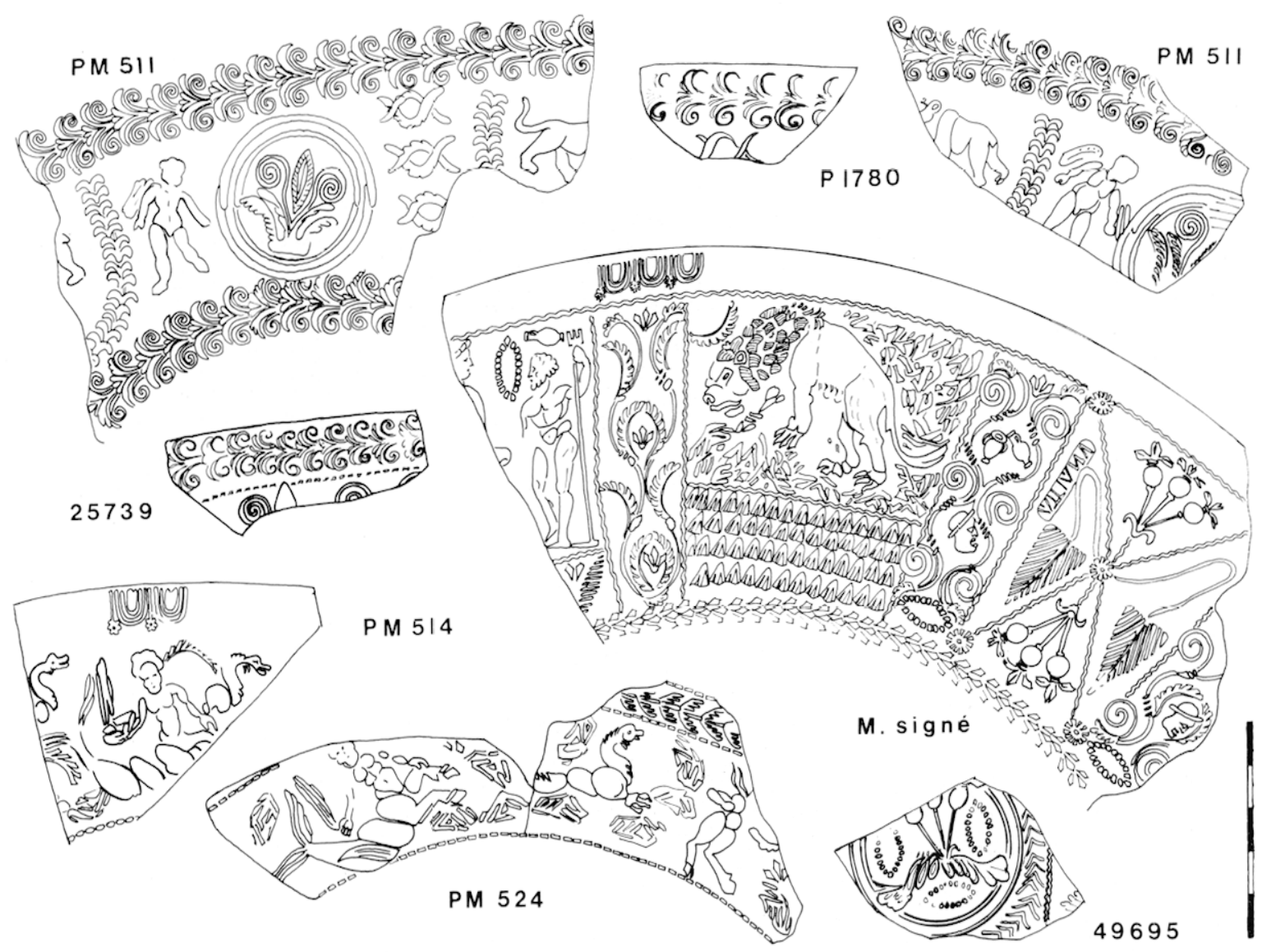

7 P-1. Style fleuri à métopes : moule signé (coll. privée). Tessons apparentés : bol (P 1780) et moules du M.A.N. (coll. Plicque); bols du M.A.N. : 25739 et 49695 (coll. Rambert).

Frises à séquences de feslons et médaillons. Ce type de construction représente une variante du décor à métopes, dans la mesure où le découpage de la surface est assuré par des éléments géométriques discontinus qui encadrent des motifs et laissent entre eux des plages libres, de forme irrégulière, où sont imprimés d'autres poinçons. La frise conservée par d'assez larges fragments de moule, PN 511 (fig. 7), porte des médaillons qui enferment une touffe végétale et des arceaux contenant tantôt un lion, tantôt un ours; les blancs sont occupés alternativement par une torsade verticale lâche et par un putto. La composition se distingue encore par l'absence d'ove et l'emploi comme bordures inférieure et supérieure de la même frise de touffes végétales. Un fragment du même décor est peut-être conservé par un fragment de bol et le même type de bordure limite une autre frise (fig. 7, 25739) dont le contenu nous fait largement défaut ${ }^{31}$.

Il apparait donc que l'analyse des structures décoratives les plus fréquemment attestées confirme les observations que permettait le répertoire (tableau II) : malgré la diversité des individus les décors à métopes se groupent en familles indépendamment 

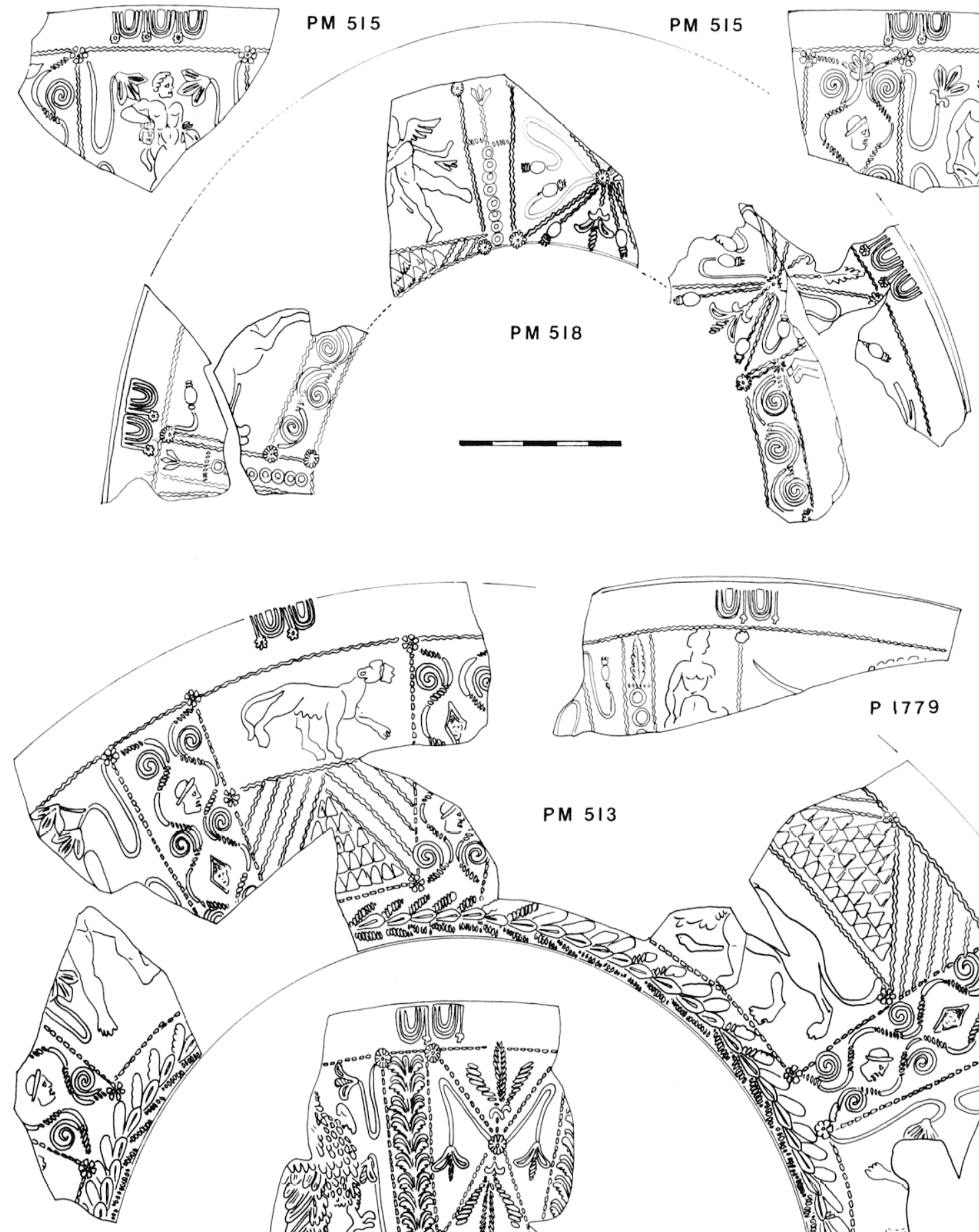

(4) (1) (1)

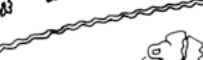

$x_{3}$
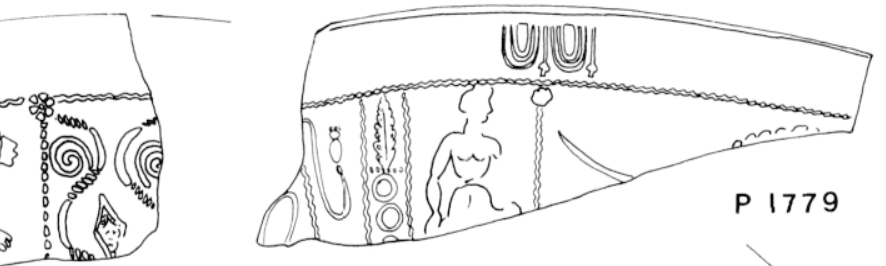

, mang
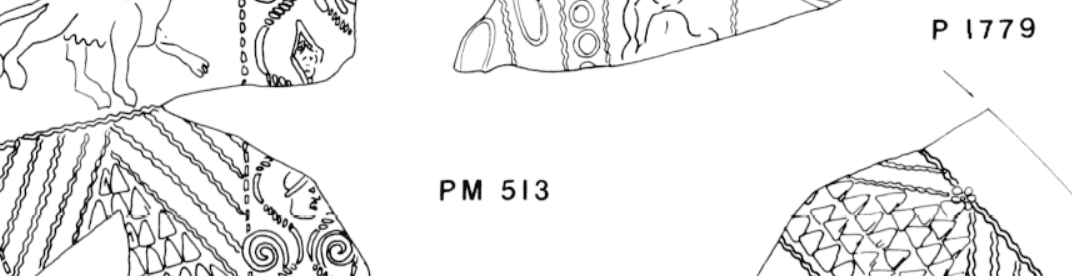

$P M 513$
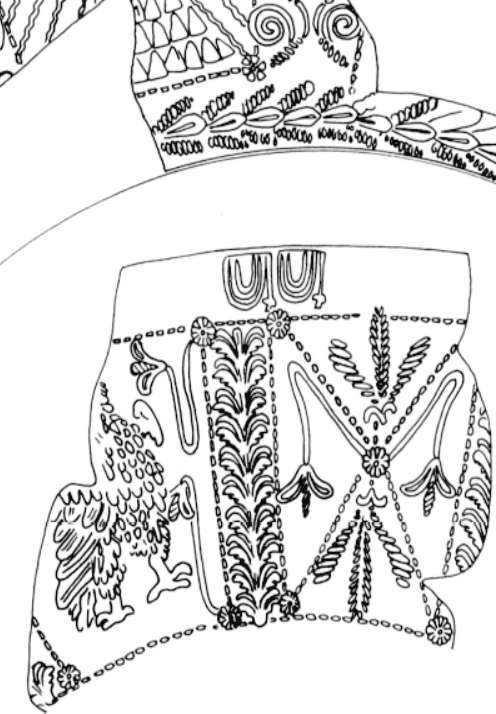

PM 519

8 P.1. Style aéré à métopes : vase (P 1779) of moules D. 37 du M.A.N. (coll. Plicque). 


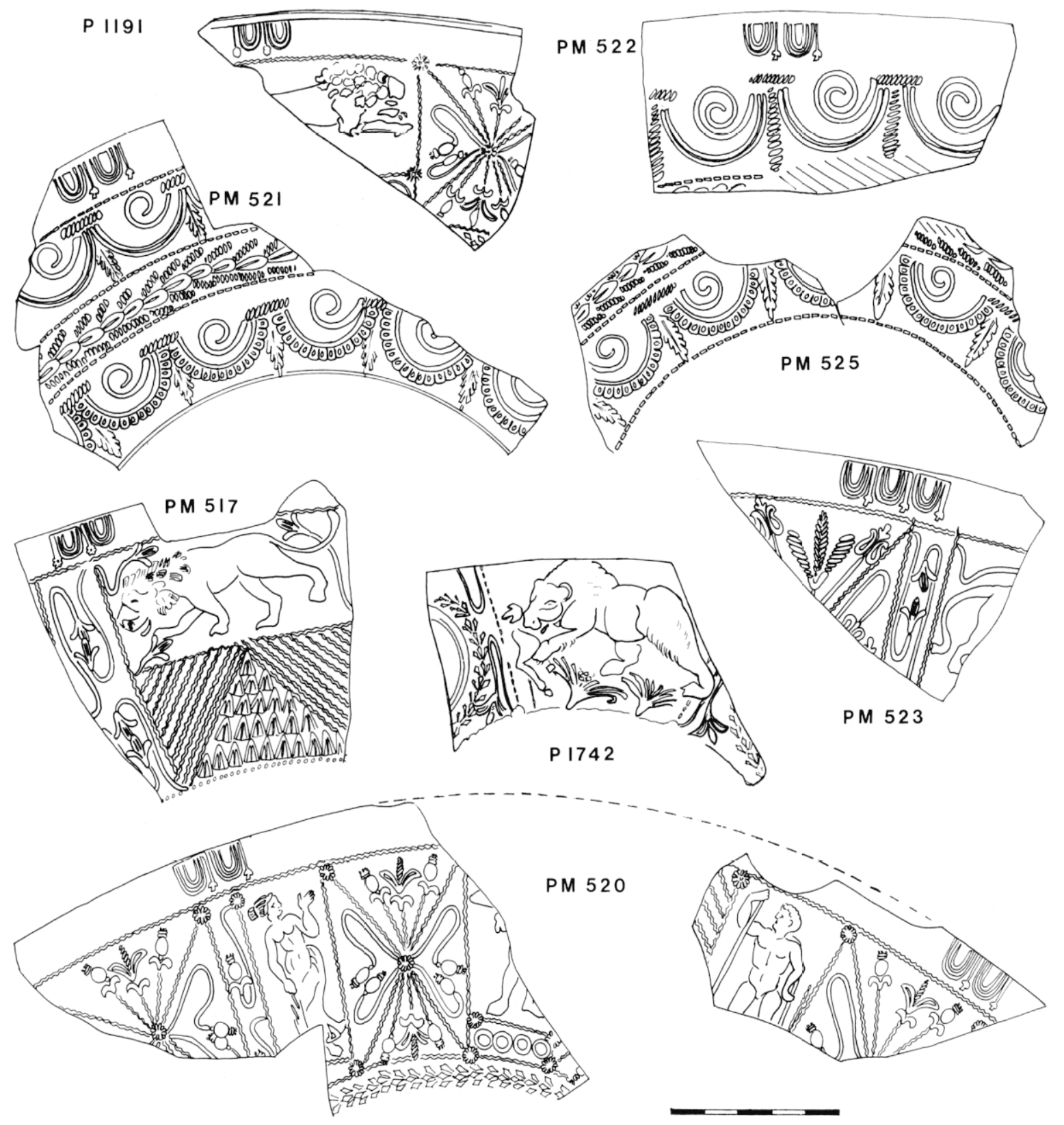

$9 \quad P$-1. Style aéré à métopes : vases (P 1191 et P 1742) et moules D. 37 du M.A.N.; tessons de moules illustrant le même répertoire, mais décorés sur deux registres (coll. Plicque).

de leur système de bordure; malgré l'existence de deux familles distinctes de compositions, il subsiste entre elles des similitudes, parfois dans la construction d'ensemble, souvent dans les motifs de séparation.

La morphologie des moules, enfin, autorise sans doute quelques remarques, mais malheureusement incomplètes. Une partie des matrices, en effet, est victime du délitage vertical des parois et les cassures de plusieurs autres nous privent de la base ou du haut de leur profil. Cependant, nous avons pu constater que les formes à métopes fleuries assez 

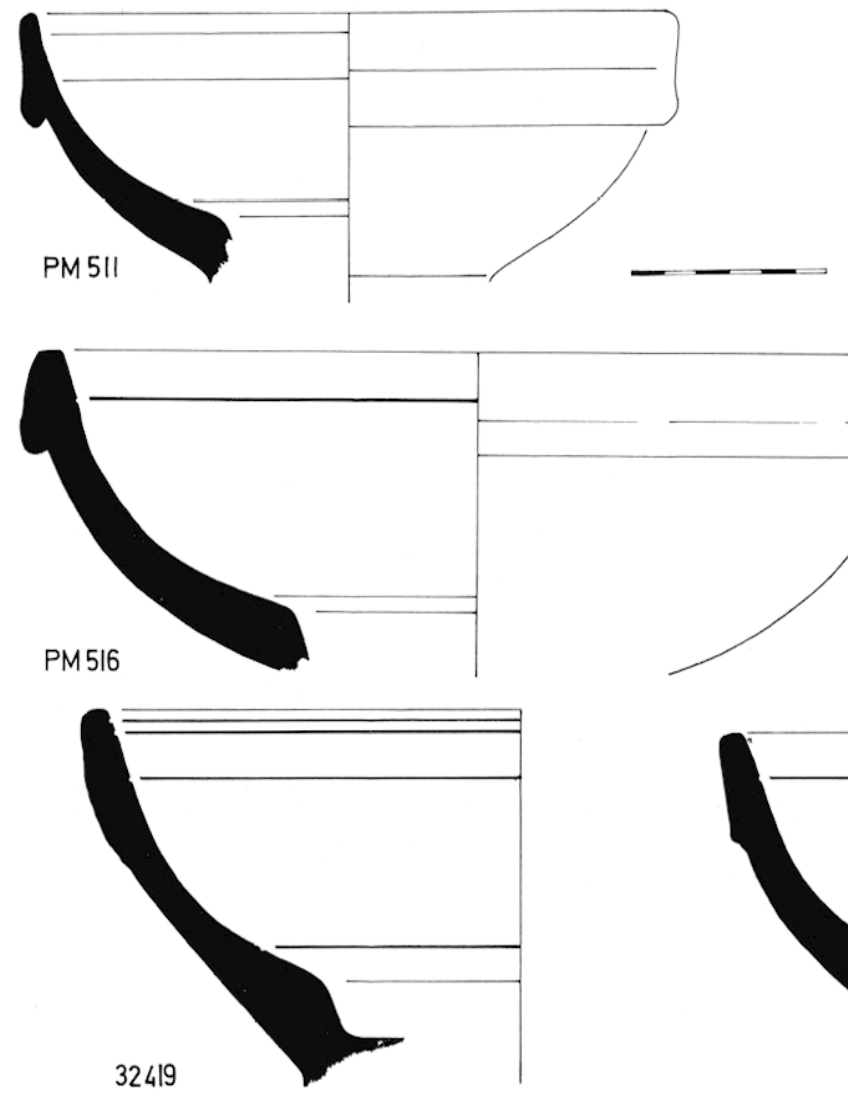

10 P-1. Moules D. 37 à bourrelet et échantillon partiellement délité illustrant le style fleuri : 32419 (M.A.N., coll. Plicque). Moule signé partiellement délité (coll. privée).

bien conservées, le moule à frise continue de monstres et la matrice à bordure végétale comportent tous un épaississement du bord à section grossièrement triangulaire, arrondi et parfois légèrement renflé à la base (fig. 10). En revanche, les décors à panneaux les plus aérés ainsi que les frises à deux registres et festons réguliers portent en général un cordon bien marqué à quelque distance du bord : peu saillant et taillé à facettes dans le second cas, nettement plus dégagé et plus arrondi dans le premier (fig. 11). Les moules conservés intérieurement sur toute leur hauteur ou en leur partie inférieure ${ }^{32}$ appartiennent sans exception au type archaïque, dans lequel est prévue une réserve d'argile destinée au pied du vase. Toutefois un échantillon à métopes aérées (PM 515), qui se distingue du reste de la collection par son bord épaissi d'un bandeau mince, haut, légèrement bombé et taillé à la base en biseau (fig. 11), ne peut être rattaché à aucun type morphologique : seule la partie supérieure du profil a subsisté.

Il apparaît ainsi que les particularités de certains supports permettent de grouper entre elles plusieurs sortes de décors, sans contredire, cependant, les distinctions que nous avons proposées entre les différentes frises à métopes. Il serait présomptueux, compte tenu 

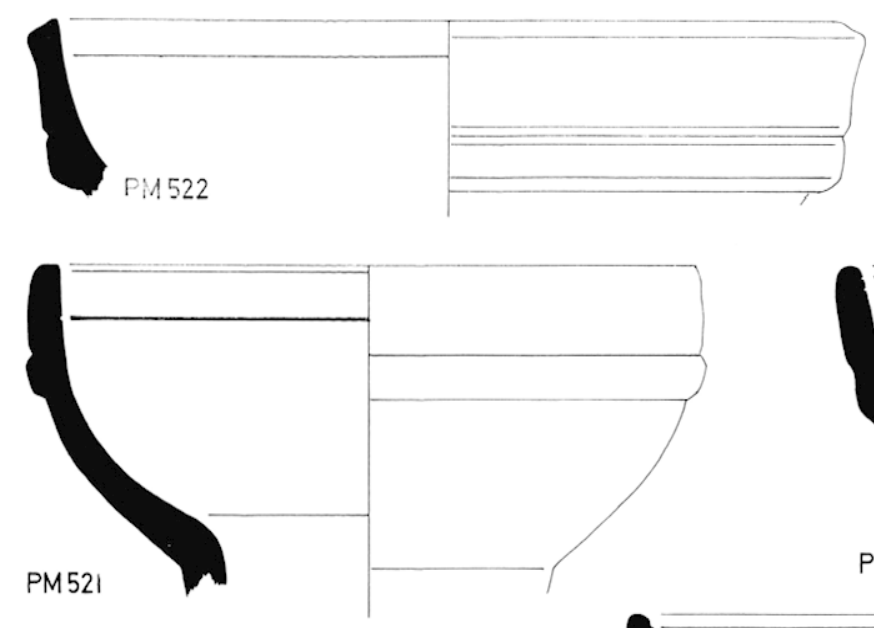

M 520
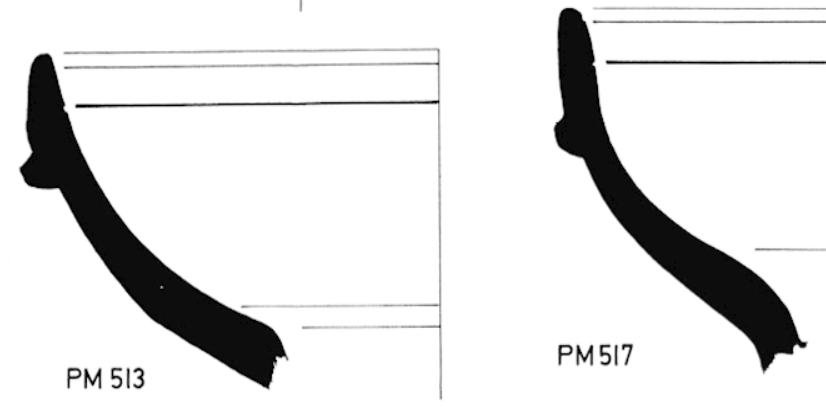

PM 519
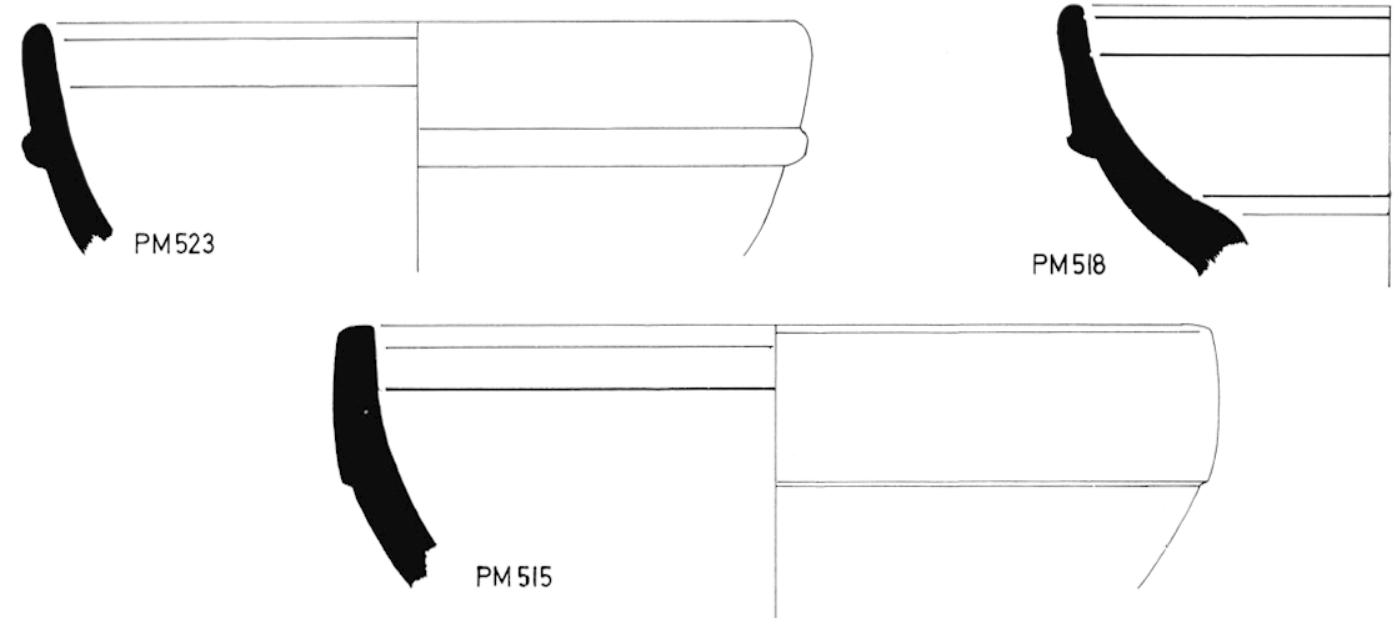

11 P-1. Moules D. 37 à cordon (décors à deux registres : P.M 521, PM 522); style aéré : PM 513, P.M 517, P.M 518, P.I 523. Moules délités : P. 519, P.I 520. Moule à bandeau : PM 515 (M.A.N., coll. Plicque).

du nombre des échantillons bien déterminés, de généraliser la portée de nos observations, mais la convergence des traits morphologiques et des caractères stylistiques tend à confirmer l'existence de groupes à l'intérieur de cette production. La variation concomitante de la forme et du décor rend moins gratuite la diversité stylistique et incite, par conséquent. à envisager des explications de ce phénomène : par exemple la multiplicité de la maind'œuvre ou la distance chronologique. 
LF DÉCOR SIGNÉ : SA PLACE DANS LE MATǴRLE $P$ - 1

III. Rapports avec les trois séries à oves B $10,203,8$

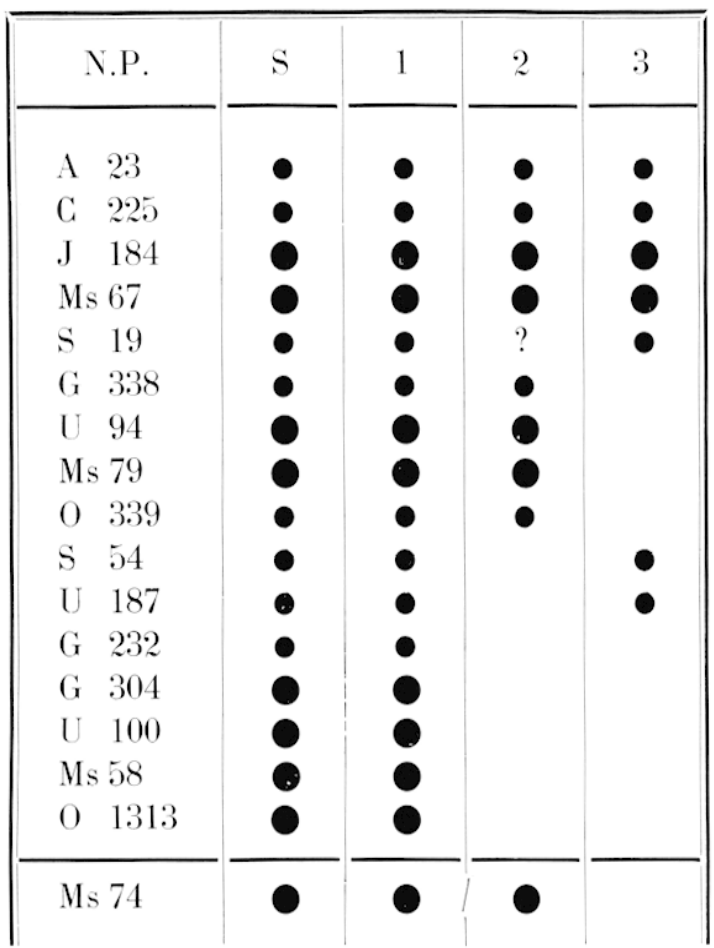

IV. Rapports avec le slyle fleuri

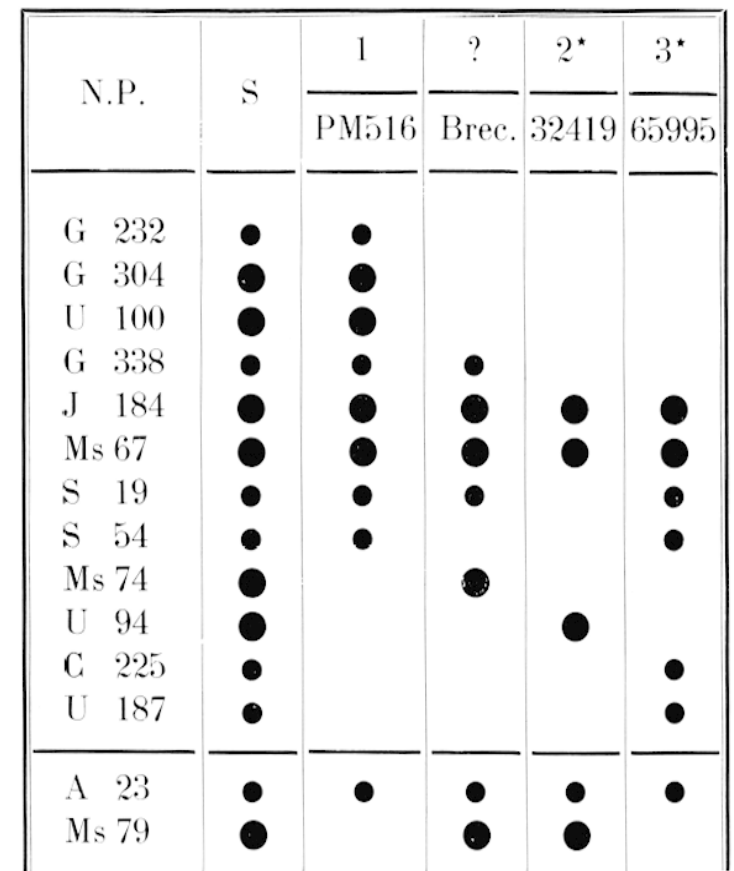

V. Rapports avec le style aéré

\begin{tabular}{|c|c|c|c|c|c|c|c|c|c|c|}
\hline \multirow{2}{*}{ N.P. } & & \multicolumn{7}{|c|}{1} & \multicolumn{2}{|c|}{2} \\
\hline & & PM515 & PM513 & PM517 & РМ518 & P1191 & PM524 & РМ514 & P1779 & PM520 \\
\hline $\begin{array}{ll}\text { O } & 339 \\
\text { O } & 1313 \\
\text { Ms } & 58 \\
\text { S } & 19 \\
\text { S } & 54 \\
\text { U } & 187 \\
\text { G } & 338 \\
\text { U } & 94 \\
\text { Ms } & 67\end{array}$ & $\begin{array}{l}0 \\
0 \\
0 \\
0 \\
0 \\
0\end{array}$ & $\bullet$ & $\begin{array}{l}0 \\
\bullet \\
\bullet\end{array}$ & • & $\bullet$ & • & 0 & & • & - \\
\hline $\begin{array}{l}\text { A } 23 \\
\text { Ms } 79\end{array}$ & $\bullet$ & 0 & ○ & $\bullet$ & 0 & $\bullet$ & & & 0 & ○ \\
\hline
\end{tabular}

Abréviations

N.P. : numéro du poinçon

1 : série portant l'ove B 10

1, ? : attribution incertaine

2 : série portant l'ove B 203

3 : série portant l'ove B 8

S : moule signé

- : poinçon tenu pour exclusif (cf. II) : exemplaire le plus complet de deux échantillons portant le mème décor 
Devant les conséquences possibles de ces disparités, il importe, désormais, de mesurer plus précisément les distances entre les différents échantillons et de définir, en particulier la place qu'occupe, parmi cux, le fragment signé, le seul qui échappe en principe à la simple détermination stylistique.

En fait, la signature $A T T I A N V$ (fig. 3 en haut), directe, en relief dans le décor paraît inédite. Elle n'a aucun rapport, en tout cas, avec les estampilles ou graffites d'un potier homonyme de Lezoux, situé par G. Simpson et J. A. Stanfield ${ }^{33}$ entre 130 et 160. Par ailleurs, le répertoire des poinçons révèle que, sur 21 motifs secondaires, la frise en partage 15 -- dont 4 inédits (fig. $5,58,67,74,79$ ) -, avec le reste des tessons attribués à $P-1^{34}$, les 6 autres (ove, palme, petits rameaux, amphorisque, tortillon fusiforme) (fig. 5, 54, 66, $68,69,73,80)$ n'étant pas encore recensés ${ }^{35} ; 2$ des 4 sujets figurés sont également communs à plusieurs bols $P$-1. D'un point de vue analytique, et si l'on élimine - comme morphologiquement mal déterminés et trop largement utilisés dans tout le style $P-1$ pour être significatifs -, le cordon ondulé et le tout petit tortillon ${ }^{36}$ (fig. 5, 5, 79), on constate l'existence de liens entre le décor signé et le contenu de seize autres frises. Les tableaux ci-joints (III-V, p. 188) montrent que ces relations intéressent entre 1 et 6 poinçons par vase, qu'elles sont globalement plus importantes avec les représentants du style fleuri à métopes et plus nombreuses, en général, avec la série 1 (ove B 10) qu'avec la série 2 (ove B 203). Du point de vue structurel la frise signée s'apparente également aux décors les plus chargés par la profusion des petits motifs, la variété de construction et de mobilier des grandes métopes, la diversité des panneaux de séparation et l'emploi, sur les fonds, de rameaux et de semis. Cependant, ces similitudes ne constituent pas un caractère exclusif : on trouve, en effet, sur ce bol, les métopes de séparation à doubles rinceaux et petits masques qui figurent dans deux décors du style sobre - PM 513 et 515(fig. 8) —, la file de menus fleurons G 338 qui borde la base de notre frise n'a, également, d'équivalent que dans un décor léger (PM 520) (fig. 9); enfin, l'emploi de poinçons exclusifs - Ms 67 et U 94 -, établit un rapport notable entre cette construction à panneaux et deux décors continus (PM 514 et 524) (fig. 7). Par ailleurs, la morphologie du moule, détérioré extérieurement vers la base du système de préhension, interdit une comparaison exacte du profil. Cependant, la place de la limite inférieure de l'épaississement par rapport au bord supérieur est plus compatible avec la hauteur des gros bourrelets triangulaires associés à plusieurs décors fleuris qu'avec celle de l'implantation des cordons qui accompagnent, le plus souvent, les métopes légères (fig. 10 et 11 ).

A ce point de notre enquête, nous voyons que le nom d'Attianus est attaché de façon particulièrement étroite à l'un des sous-groupes de ce que nous appelons le style $P-1$ : celui que caractérisent les métopes foisonnantes, mais que le décor qui porte la signature entretient, plus que d'autres de la même catégorie, des relations avec un autre sous-groupe :

33 O.c., p. 165-169 et pl. 85-87; deux estampilles intra ou infradécoratives et rétrogrades (sur vases) : $O F$. ATT et ATTIANI.O et plusieurs gramites infradécoratifs, dont le plus complet (pl. 87, 21) se lit ATTIANI.

34 A 23, C 225, G 232, G 304, G 338, J 184, S 19, S 54, U 94, U 100, U 187, Ms 58, Ms 67, Ms 74, Ms 79.

35 C. n. 16 .

36 A 23 et is 79. 
VI. Relations entre le style fleuri et le reste des décors $P-1$

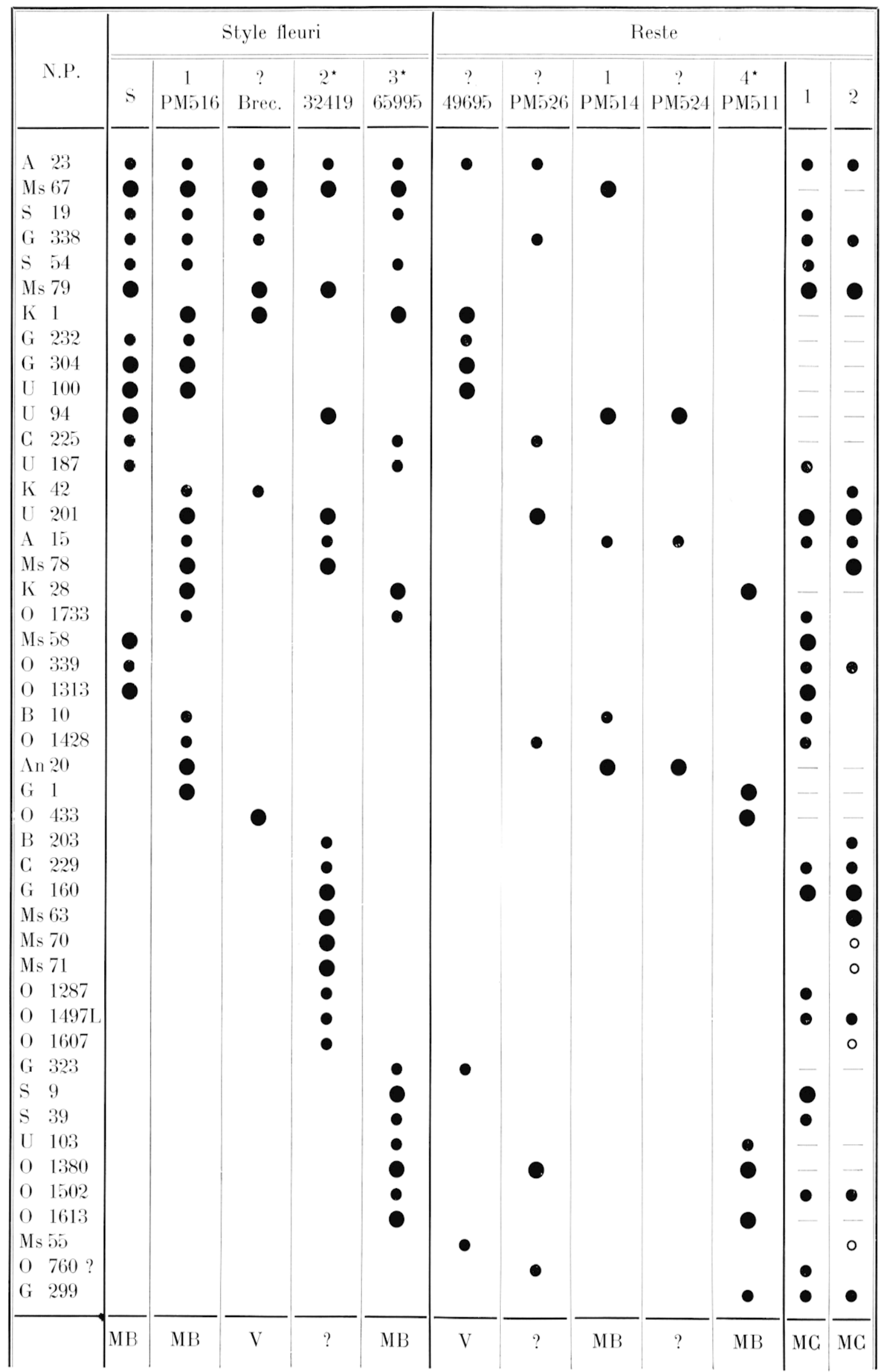


celui des constructions sèches, aérées et, à différents égards, plus banalement répétitives. Ainsi, par ses structures et son répertoire la frise d'Allianus paraît un garant essentiel de l'homogénéité de $P-1$.

L'extension des comparaisons de répertoire à l'ensemble des décors fleuris - moules PM 516 et 512, MAN 32419 et 65995, vases 1791 et de Brecon, - entraîne plusieurs constatations. Ainsi, le reste du matériel $P-1$ se décompose en deux groupes (A et B). Le premier comprend les derniers moules à bourrelet, PM 514 et 511 , trois fragments comparables à ces derniers par leur décor - PM 524, tesson de matrice informe très voisin de PM 514, MAN 25739 et P 1780 (petits morceaux de vases sans oves, de la famille de PM 511), le petit tesson de bol 49695 et le fragment de moule PM 526, d'attribution douteuse, mais assez proche des frises fleuries. Le second réunit tous les moules à bandeau ou cordon — PM 513, 515, 517, 519, 521-523 - , 3/4 morceaux de vases à décor aéré - $\mathrm{P} 1191$, 1742, 1779, 1777? - , un moule délité de même style (PM 520) et un tesson de matrice (PM 525), comparable par sa frise à PM 521. Le premier groupe entretient avec le style fleuri un nombre appréciable de relations exclusives, alors que ces rapports sont beaucoup plus rares avec le second groupe (G 299, Ms 55, 0760 ?). Enfin, la quasi-totalité des motifs communs au style fleuri et à A et B n'apparaissent dans le groupe A que sur le tesson PM 526 : s'il ne faut pas exclure ce moule du matériel $P$-1, cette particularité s'explique vraisemblablement par sa parenté avec les décors fleuris. Par ailleurs, l'ensemble du groupe $\mathrm{B}$ (style aéré, moules à cordon ou bandeau) conserve avec le répertoire du style fleuri à panneaux de nombreux points communs : une trentaine d'éléments iconographiques, en effet, apparaissent à la fois dans les deux séries. Le tableau VI (p. 190), réduit, pour le style fleuri et le premier groupe, aux échantillons les plus complets, pour le second ensemble, aux familles déterminées en fonction des oves, met en évidence ces particularités essentielles : d'une part l'abondance des liens entre le style surchargé el le style léger et le détail des décors intéressés, d'autre part la coïncidence d'une morphologie - celle des moules à bourrelet - avec l'emploi d'éléments de répertoire actuellement exclusifs, quelles que soient, par ailleurs, les structures décoratives (géométriques ou continues) dans lesquelles le potier les a intégrés.

Dans l'état actuel de notre documentation, l'existence, à l'intérieur du matériel attribué à $P-1$, de groupes stylistiques et, pour les moules, morphologiques, demeure donc peu contestable. Cependant, l'étroitesse des relations que nos dernières analyses semblent

\section{Sigles et abréviations}

N.P. : numéro du poinçon série portant l'ove B 10 série portant l'ove B 203

série portant l'ove B 8

série à bordure végétale

moule signé

exemplaire le plus complet de deux

échantillons portant le même décor
○ : tesson privé de bordure

- : poinçon tenu pour exclusif ( $\mathrm{cf}$. II)

MB : moule à bourrelet

$\mathrm{MC}$ : moule à cordon

$\mathrm{V}$ : vase

? : classement impossible 
avoir fait ressortir entre les deux séries, correspondant, chacune, à une variété principale de matrice, incite à expliquer les différences entre elles soit par l'évolution de la technique d'un même individu ${ }^{37}$, soit par la collaboration assidue de deux potiers, ou la succession directe -.. passablement servile - de l'un à l'autre.

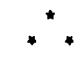

Pour situer un style nous disposons de plusieurs moyens : le nom du potier, lorsqu'il est déjà connu et daté, les qualités propres des décors, la découverte en stratigraphie de témoins iconographiques de l'atelier.

En la circonstance, la datation par le nom aboutirait à une erreur : on connait déjà un Attianus, mais il s'agit apparemment d'un simple homonyme. En effet, les produits de ce potier diffèrent totalement de notre matériel par le contenu et l'organisation des décors et, comme nous l'avons dit, aucune des marques qui accompagnent ces derniers ne coïncide avec celle de notre tesson signé ${ }^{38}$. Restent donc les références internes : la qualité du style et les conditions de découverte de nos échantillons. Les observations que nous avons déjà faites sur l'iconographie de ce matériel conduisent logiquement à deux conclusions : le groupe $P-1$ témoigne de l'influence probable des décors flaviens de la Gaule du sud tout en se rattachant, par le détail des structures et le répertoire, aux ateliers de Gaule centrale datés entre la fin du $\mathrm{i}^{\mathrm{er}}$ siècle et le premier quart du $\mathrm{II}^{\mathrm{e}}$. L'influence des compositions méridionales -.. essentiellement de l'époque de Vespasien --, fréquemment observée au début de l'expansion des officines du Centre, se manifeste ici par certains usages dans l'organisation des motifs : emploi de sautoirs composites exclusivement végétaux et constitués principalement de feuilles, fleurons et boutons reliés au centre de la métope qui les enferme par de longues tiges minces, souples ou raides ${ }^{39}$; fréquence des "feuilles volantes "40 -.. petits poinçons végétaux ou tortillons servant au remplissage et attachés aux cordons ou aux angles des panneaux par un pétiole sinueux --, des triangles ou rectangles d'imbrications, des bordures de fleurons ou chevrons à la base des décors, des motifs ou structures complexes comme les médaillons arboréidés ou les "festons alternants $»^{41}$. On notera aussi les compositions à deux registres, héritées dans le Centre comme dans le Sud de la décoration des bols carénés. L'essentiel des ressemblances s'arrête là, cependant, car le répertoire entretient des rapports presque exclusifs ${ }^{42}$ avec les ateliers du Cientre. Ainsi $X-O$, potier inédit, contemporain de $X-1$, ou de peu antérieur, partage avec $P-1$ au moins 10 poinçons secondaires et 3 poinçons principaux; toutefois ce que

37 D'une façan générale on observe que le style des décors du Centre, à partir du règne de Trajan, tend à s'alléger et à se simplifler, mais le détail de cette évolution reste à préciser.

38 Cf. par exemple : F. Hermet, La Graufesenque, Paris, 1934, II, pl. 29, 8, pl. 33, 14, 15, 18.

39 Seule la lionne 01502 parait dans le répertoire du second Attianus; pour ses signatures, cf. supra, n. 33.

40 F. HeRmet, o.c., p. $185,6^{\circ}$.

41 Ibid., pl. 84, 10 : festons habités séparés par des plages meublées de personnages.

42 Trois types figurés, cependant, sont attestés dans le Sud : O 28, O 580, oiseau palinscope (O 2290-2299); quelques poinģons secondaires y ont aussi des analogues: G 299 (НЕRмet, l.c., pl. 13 B, ,54), G 304 (ibid., 43 ), Ms 64 (ibid., 9), par exemple. 
nous connaissons des décors ne permet guère de comparaisons de structure ${ }^{43}$. Le polier à la Roselte utilise seulement 9 des motifs de $P-1: 3$ décors secondaires et 6 sujets figurés ${ }^{44}$. En revanche on trouve chez lui un répertoire de types iconographiques parallèle à celui de notre potier : corbeille d'acanthes (K 2), arceau festonné (F 75), palme ( $\mathrm{J} 127)$, chevron spiralé de feuilles découpées (G 345) - pour ne citer que les éléments les plus remarquables. Les deux décorateurs se ressemblent aussi par quelques procédés d'organisation partiels : colonnes de fleurons ou d'anneaux entre les panneaux principaux, feuilles volantes, association de la touffe végétale $\mathrm{G} 1$ et d'une corbeille d'acanthes, métope coupée, meublée en haut d'un animal (parfois le même ours), en bas d'un triangle isocèle d'imbrications, reposant sur sa base et encadré de deux triangles striés de cordons ondulés ${ }^{45}$. D'autres potiers datés du règne de Trajan participent, à un titre moindre, du répertoire $P-1: X-1$, $X-11$ et $X-13$ surtout $^{46}$. Et l'on retrouve également chez divers décorateurs de cette époque quelques-unes de ses méthodes de composition : petits poinçons de remplissage en semis ( $X-1, X-2, X-3 /$ Drusus $I, X-4$, polier à la Rosette...) ou accrochés aux cordons de séparation $(X-3 /$ Drusus $I)$, colonne d'anneaux surmontée d'un fleuron $(X-12)^{47}$. Dans l'ensemble, ce groupe de décorateurs fait preuve d'une telle imagination dans l'invention des petits motifs, d'une telle variété dans la surcharge iconographique qu'on ne peut espérer pousser plus loin les comparaisons ponctuelles entre $P-1$ et tel d'entre eux, mais c'est incontestablement du même esprit que relèvent toutes ces créations. Toutefois, leur individualisme apparent et l'absence de chronologie fine des céramiques de cette époque nous interdisent de dater par référence les sous-groupes de $P-1$ et, en particulier, de défendre valablement l'hypothèse d'une évolution interne ${ }^{48}$.

En ce qui concerne les critères archéologiques de datation, nos ressources sont maigres. Tous les tessons, en effet, à l'exception du moule signé et du fragment de bol de Brecon, proviennent de la collection Plicque ou, pour MAN 25739 et 49695 , de la collection Rambert : les conditions précises de leur découverte demeurent donc inconnues. L'échantillon signé a été acheté près des Eyzies. Le vendeur, qui le tenait lui-même d'un précédent propriétaire, le présentait comme d'origine locale ${ }^{49}$. Mais, faute de preuve démonstrative, il paraît actuellement injustifié de s'interroger sur les raisons de la présence, dans un

43 A 14, A 23, B 8, C 225, G 298, G 338, S 19, S 54, U 93 ?, U 187; O 723, O 1733, O 2292. Les quelques compositions que nous connaissons par des moules ou bols se caractérisent principalement par des systèmes à arcatures.

$44 \Lambda 1, A 23, \mathrm{G} 1 ; 012,028,0723,01497 \mathrm{~L}, \mathrm{O} 1607, \mathrm{O} 2292$.

45 Fleurons (S.S., pl. 20, 252); anneaux (ibid., pl. 23, 286, 287); feuilles volantes (ibid., pl. 21, 261, 262 ; pl. 22, 267); G 1 et acunthes (ibid., pl. 22, 278, 281); métopes coupées (ibid., pl. 20, 253, 255; pl. 22, 2r6; pl. 23, 286).

$46 X-1$ : A 23, G 232, G 323, U 187, O 723, O 860 ; X-11/Ioenalis : A 1, C 229, F 74, G 299, L 103, O 1733 ; $X-13$ !Donnaucus : A 1, C 229, F 74, G 323, U 103, O 28.

47 Cf. S.S., semis $(X-1:$ pl. 1 et 2 passim; $X-2$ : pl. 4-6 passim; $X-3:$ pl. 10, 11, 13, 14 passim ; $X-4:$ pl. 19, 242 ; p. à la Rosette : pl. 20, 21, 23-26 passim) ; motifs suspendus $(X-3:$ pl. 10, 124, $125 ; 13,156)$; colonne d'anneaux $(X-12$ : pl. 35, 413).

48 Les formes des moules confirment globalement la vraisemblance de cette chronologie, mais nous nous sommes heurtés à la même difficulté pour expliquer la diversité iconographique et morphologique dans un autre atelier de l'époque de Trajan (Liberlus (ou Liberti ?) I: les premiers slyles à eslampilles, dans Gallia, 36, 1978, p. 89-141.

49 La recherche qu'a bien voulu faire $\mathbf{~ I . ~ H . ~ B r u n ~ n o u s ~ a ~ p e r m i s ~ d ' a p p r e n d r e ~ q u e ~ l ' a n t i q u a i r e ~ a c q u e ́ r e u r ~ l ' a v a i t ~}$ acheté vers 1973-1974 dans une vente publique et que l'objet provenait alors de la collection du curé de Villeréal (Lot-et-Garonne). 
contexte antique du Sud-Ouest, d'une matrice décorée dans la tradition de la Gaule centrale : il n'est pas exclu qu'il s'agisse simplement d'un objet de collection arrivé là récemment et dans des circonstances fortuites. Quoi qu'il en soit, les conditions exactes de la découverte de cet échantillon nous font également défaut. Le vase de Brecon, décrit. par F. Oswald ${ }^{50}$, n'est datée que par l'abandon du site vers 140 .

L'expérience présente aboutit à plusieurs conclusions. Elle fournit, d'abord, la première documentation critique sur un atelier inédit, baptisé provisoirement $P-1$, et permet occasionnellement de préciser les informations sur un style voisin, peu à peu distingué de $P-1$ et que nous avons nommé $P-4$. Nous connaissons aussi le responsable au moins d'une partie des produits attribués à $P-1$ : il s'appelle Altianus, mais ce n'est qu'un homonyme du potier déjà bien attesté et situé entre 130 et 160. En effet tout différencie les deux séries de décors; de plus, les ressemblances morphologiques et les comparaisons stylistiques concourent également à dater l'essentiel de cette production de $P$ - 1 entre la fin $d_{u} \mathrm{i}^{\mathrm{er}} \mathrm{s}$. et le début du règne d'Hadrien. L'environnement archéologique d'un des vases s'accommode, sans apporter plus de précision, avec cette chronologie. Il convient donc, désormais, de distinguer, comme on l'a fait pour Drusus, Paternus, Secundinus, Seruus, des styles homonymes : Altianus $I / P-1$ et Altianus $I I /$ ancien Attianus.

Pour positifs qu'ils soient, ces résultats ne sauraient être considérés comme définitifs. Le matériel tenu pour le plus sûr est encore peu nombreux et la richesse de quelques échantillons en poinçons inédits ou employés une seule fois autorise à supposer que nous ne connaissons qu'une petite partie des décors $P_{-} 1$ : de nouvelles trouvailles multiplieraient sûrement les relations entre les décors, mais accroîtraient peut-être encore le répertoire de l'atelier. I'autre part une frange d'incertitude subsiste autour du groupe principal : nous avons signalé comme douteux un ou deux fragments isolés et écartés sans l'attribuer à quiconque, un petit groupe de lessons. L'extension de l'enquête permettra de dissiper ces doutes, mais il nous paraît déjà très probable que quelques-uns de ces échantillons témoignent de la diversité de la production de l'époque de Trajan, dont les quelques officines déjà connues et publiées incitent sans doute trop facilement à méconnaitre la richesse.

Par ailleurs, la constitution d'ensembles stylistiques, le plus souvent anonymes, pose par elle-même des problèmes de fond : ceux que soulèvent le choix des critères d'homogénéité des groupes et l'interprétation d'éventuelles disparates à l'intéricur de séries cohérentes d'un autre point de vue. Nous nous sommes fondés sur trois critères principaux : le répertoire des poinçons - envisagés surtout dans leurs associations et en fonction de leur rareté -, les structures décoratives - considérées à la fois globalement et dans le détail des motifs composites et des petits agencements --., enfin, la morphologie des moules. Et le déroulement de notre étude nous a conduits à nous interroger sur l'intérêt spécifique de chacun d'eux.

50 Dans R. E. M. Whesier, The Roman Forl near Brecon, Londres, 1926. L'étude de la sigillée (T. D. Pryce. el F. Oswald, p. 122-213) n'autorise pas à dissocier le lot auquel appartient notre tesson (no 118, p. 165, fig. 76) de l'ensemble des vases correspondant à la première occupation du site (ibid., chronologie p. 69-74). Ce groupe comprend quelques échantillons du Sud (Flaviens-Trajan, nos $31,47,62,75,79)$ et trois fragments de Galule centrale (no 118 : P-1, 121 : Quintilianus (G), 123 : Docilis (S)). 
L’inventaire des poinçons est le moyen le plus éprouvé de définition des styles. Nous avons constaté, encore une fois, que le nombre des éléments associés importe souvent plus que la présence d'un motif tenu hypothétiquement pour exceptionnel et qu'au début d'une enquête l'honnêteté commande de remettre souvent en question l'attribution des petits tessons. En particulier, il est apparu que l'importance des oves, considérés souvent comme des indices déterminants, devait ètre pondérée par la multiplicité cohérente des décors qui les accompagnent. Enfin, nous avons été amenés à nous demander s'il était vraisemblable que le même potier emploie deux ou plusieurs poinçons, bien individualisés par leur complexité, relevant exactement du même type et remplissant les mêmes fonctions, mais distingués par de menues différences de proportions ou de morphologie ${ }^{51}$. L'expérience semble prouver que ces doublets caractérisent bien souvent des styles différents, mais le problème n'a jamais été abordé de front et il reste à multiplier les observations pour démontrer si, oui ou non, ces poinçons parallèles peuvent, à eux seuls, prouver l'existence de styles distincts et constituer un instrument immédiatement efficace de discrimination.

L'analyse des structures iconographiques n'a, jusqu'à ces dernières années, fait qu'accidentellement l'objet d'études systématiques et elle a été plus rarement encore utilisée dans la définition des styles individuels. Encore l'a-t-on réduite souvent, dans ce cas, à la notation de telle particularité isolée, tenue, d'ailleurs à juste titre, pour une habitude du potier, donc un indice significatif. Dans le cas présent, nous avons observé qu'il pouvait y avoir une opposition entre les attributions fondées sur le répertoire et celles qu'on étaie sur des constructions et agencements peu fréquents : nous avons estimé, sous réserve de vérification, qu'une structure se prêtait aisément à la copie et n'indiquait qu'une parenté entre deux frises, lorsque l'outillage décoratif ne confirmait pas - directement ou indirectement - leur attribution au même artisan. Par ailleurs, l'examen méthodique et détaillé des compositions, combiné à l'inventaire critique du mobilier a révélé la présence, dans le style $P-1$, de deux séries principales, qui actuellement n'ont en commun qu'une part importante, mais limitée, du répertoire total des poinçons. Dans la mesure où une mise en œuvre particulière est liée - comme nous l'avons montré par cet exemple et ailleurs ${ }^{52}--$ à un outillage partiellement original elle fournit donc des indices non négligeables surtout pour la définition de styles très voisins.

Enfin, la typologie des moules reste encore un moyen peu exploité de classament, même pour les fouilleurs d'ateliers. En la circonstance, la division de P-1 en deux sousgroupes stylistiques coïncide avec l'existence de deux formes principales de moules ${ }^{53}$. Bien plus, le groupement expérimental des échantillons d'après leur morphologie a permis de découvrir une communauté exclusive de répertoire entre des frises qui s'organisent selon des principes trop différents pour suggérer au premier abord une réelle parentét ${ }^{54}$. Cet exemple tend donc à suggérer une hiérarchie entre les critères choisis : la forme des

51 Les différences de tailles sont souvent plus difficiles à apprécier et nous connaissons des exemples _- très rares - d'emploi par le même potier d'un poinçon el de sa copie réduite.

52 Cf., par exemple, les styles A et C de Liberius (Gallia, 36, 1978, p. 115, 118,119 et 135 .

53 I.e moule à bandeau PM 515 reste pour le moment un apax incomplet et son décor est trop mutilé pour permettre de déterminer la signification de cette difference.

54 Cf. supra, p. 191 el tableau VI ; voir également les decors a festons ef le style aéré. 
moules et le mobilier qui les orne constituent ... au moins pour les ateliers du Centre que nous connaissons - des moyens primordiaux et indissociables d'identification des styles, alors que la fantaisie du décorateur introduit, normalement, dans la mise en ourre une multiplicité de schémas d'ensemble, que nous ne maîtrisons que plus lentement.

Cependant, l'existence de sous-groupes, là où l'étıde du seul répertoire ne donnerait qu'une idée globale de la production de $P$-1, soulève quelques difficultés, même s'il n'est pas question de partager les échantillons entre deux ateliers. Nous constatons, d'abord, que l'association répétée d'une morphologie, d'un répertoire et d'une mise en ouvre particuliers ne fournit aucun argument en faveur de l'idée trop facilement reçue d'un partage d'attributions entre un fabricant de moules nus et un décorateur ${ }^{55}$. D'autre part, pour organisée et cohérente qu'elle paraisse désormais, la diversité des moules reste insuffisamment expliquée : nous manquons, en effet, du classement chronologique des formes qui nous permettrait dans le cas présent de mesurer la valeur respective des deux hypothèses qui rendraient compte de la variété du matériel attribué à $P-1$ - la multiplicité des créateurs de moules de l'atelier, l'évolution diachronique de la production.

Colette BÉmont, George Rogers.

55 Lees mêmes conclusions se digagent de nos recherchess antérieures. Cf. en particulier Moules de gobelets ornés..., passim, Libertus (ou liberti ?, dans Ciallia, 36, 1978, I.c. el 37, 1979, p. 180-181, Moules el slyles: cssai de mélhodologie, dans Rei cretariae romanae fautorum Acla XIX-XX, 1977 (1979; , p. 5-24. 